\title{
Recent advances on crystalline materials-based flexible memristors for data storage and neuromorphic applications
}

\author{
Yang $\mathrm{Li}^{1 \dagger^{*}}$, Cheng Zhang ${ }^{1 \dagger}$, Zhiming $\mathrm{Shi}^{1}$, Chunlan $\mathrm{Ma}^{1^{*}}$, Jun Wang ${ }^{1}$ and Qichun Zhang ${ }^{2,3^{*}}$
}

\begin{abstract}
Memristors have recently emerged as promising contenders for in-memory computing and artificial neural networks, attributed to their analogies to biological synapses and neurons in structural and electrical behaviors. From the diversity level, a variety of materials have been demonstrated to have great potential for memristor applications. Herein, we focus on one class of crystalline materials (CMs)-based flexible memristors with state-of-the-art experimental demonstrations. Firstly, the typical device structure and switching mechanisms are introduced. Secondly, the recent advances on CMs-based flexible memristors, including 2D materials, metal-organic frameworks, covalent organic frameworks, and perovskites, as well as their applications for data storage and neuromorphic devices are comprehensively summarized. Finally, the future challenges and perspectives of CMs-based flexible memristors are presented.
\end{abstract}

Keywords: flexible memristor, 2D material, metal-organic framework, covalent organic framework, perovskite

\section{INTRODUCTION}

In 1971, on the basis of fundamental electric circuit theory, Chua [1] predicted that there should exist the fourth basic passive circuit element of memristor beyond the known resistor, capacitor and inductor, in order to complement the functional relations between the charge and the magnetic flux (Fig. 1). He demonstrated that the memristor was a nonlinear resistor, where the resistance value could vary with the history of the current or voltage input. In other words, the memristor can remember the flowing charge or magnetic flux by the change of the resistance, which is short for the words of "memory" and "resistor". However, although the theoretical prediction is revolutionary, the development of proof-of-concept memristors remains slow until the first memristor device of $\mathrm{Pt} / \mathrm{TiO}_{2} / \mathrm{Pt}$ was successfully fabricated by Hewlett Packard Laboratory in 2008 [2]. Since then, memristors have been ushered into a period of rapid advancement and aroused tremendous attention in both academic and industrial communities [3-7].

Recently, memristors have emerged as promising contenders for next-generation high-capacity information storage and computing systems, attributing to their advantages of fast data transfer rate, short access time, low power consumption, and the compatibility with complementary metal-oxide-semiconductor (CMOS) technology [8-14]. More importantly, they have exhibited great potential in the applications of nonvolatile memory, logic computing and brain-inspired neuromorphic hardware [15-22]. These three interrelated technologies provide a feasible route for developing a novel in-memory computing architecture that integrates information storage and processing in one system $[12,13]$, which can break through the existing von Neumann bottleneck and memory wall of traditional computing systems. A typical memristor device generally composes of two electrodes and a switching layer between them, which can switch

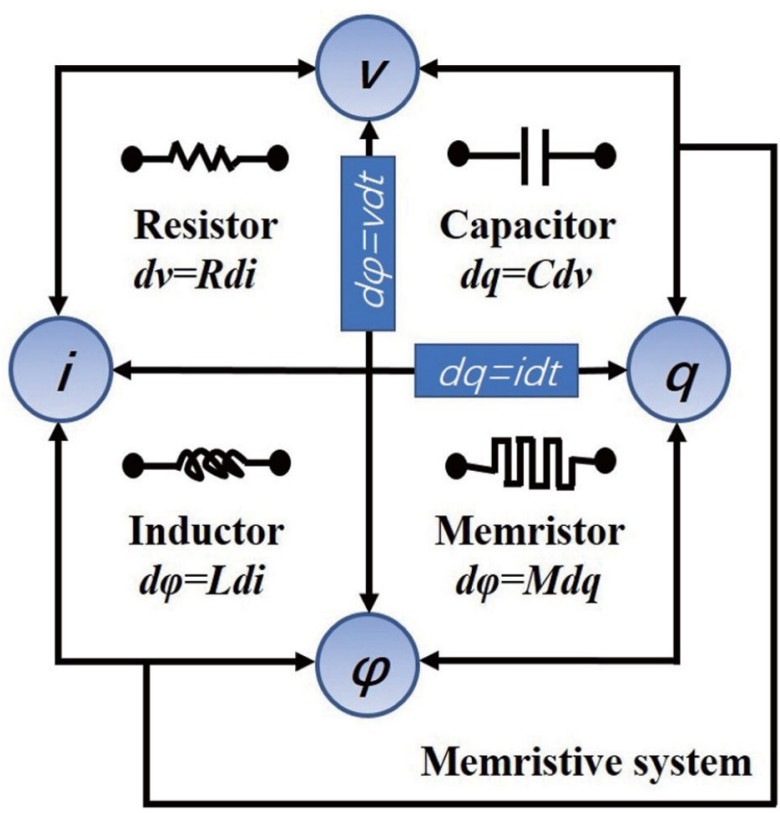

Figure 1 A schematic illustration of the four fundamental two-terminal circuit elements of the resistor, capacitor, inductor and memristor, as well as the relations between the circuit variables.

\footnotetext{
${ }^{1}$ Jiangsu Key Laboratory of Micro and Nano Heat Fluid Flow Technology and Energy Application, School of Physical Science and Technology, Suzhou University of Science and Technology, Suzhou 215009, China

${ }^{2}$ Department of Materials Science and Engineering, City University of Hong Kong, Kowloon, Hong Kong SAR 999077, China

${ }^{3}$ Center of Super-Diamond and Advanced Films (COSDAF), City University of Hong Kong, Hong Kong SAR 999077, China

$\dagger$ These authors contributed equally to this work.

* Corresponding authors (emails: liyang@usts.edu.cn (Li Y); wlxmcl@usts.edu.cn (Ma C); qiczhang@cityu.edu.hk (Zhang Q))
} 
between high and low resistance states (RSs) in response to an external electric voltage. The high RS (HRS) can function as "0" code in a binary system, and thus the switching from HRS to the low RS (LRS) can be equivalent to "0"-to-" 1 " binary conversion. Based on this concept, it is reasonable to anticipate multilevel storage (e.g., "0", "1", "2") when more than two resistive states of one memristor device can be triggered under the electric stimuli $[23,24]$.

To date, a variety of functional materials have been applied for memristor applications [25-29], including organic materials [30-32], inorganic compounds [33-35] and organic-inorganic hybrid materials [36-38]. In this review, we pay attention to a special class of materials: flexible crystalline materials (CMs). Attributed to their low defect density, long-range ordering, and potential flexibility, flexible CMs have been well documented to be ideal platforms for achieving top-performing optoelectronic devices and circuits [39-41]. Highly ordered and purposively patterned CMs-based semiconductors hold great potential for practical applications, since they not only warrant device uniformity with selective charge transport, but also restrain the cross-talk problem in the devices $[42,43]$. Therefore, the scaling up of aligned and patterned CMs-based devices has been regarded as one appealing field for integrated circuits. During the past decades, a great success has been witnessed for CMsbased high-performance flexible optical and electronic devices
$[39,44]$. Moreover, CMs have shined with extraordinary splendors in the development of memristive materials and devices. This review focuses on the recent research progress about CMsbased flexible memristors, including two-dimensional (2D) materials, metal-organic frameworks (MOFs), covalent organic frameworks (COFs), and perovskites, as illustrated in Fig. 2. The challenges and outlook for the further optimization of CMsbased flexible memristors are also summarized.

\section{MEMRISTOR STRUCTURE AND SWITCHING MECHANISMS}

\section{Memristor device structure}

\section{Vertical memristor device structure}

Many reported CMs-based flexible memristors are fabricated with a vertical metal-insulator-metal (MIM) configuration. The vertical MIM structure sandwiches the active layer between the bottom and top electrodes, which can form regular device cell arrays such as a crossbar geometry (Fig. 3a) [49]. Such vertical structure can help to scale down the thickness of active layer towards sub-10 nm, affording a lower operating voltage and higher integration density, which is a superior merit compared with the lateral configuration. In addition, it facilitates the vertical stacking of different active layers for heterostructures,

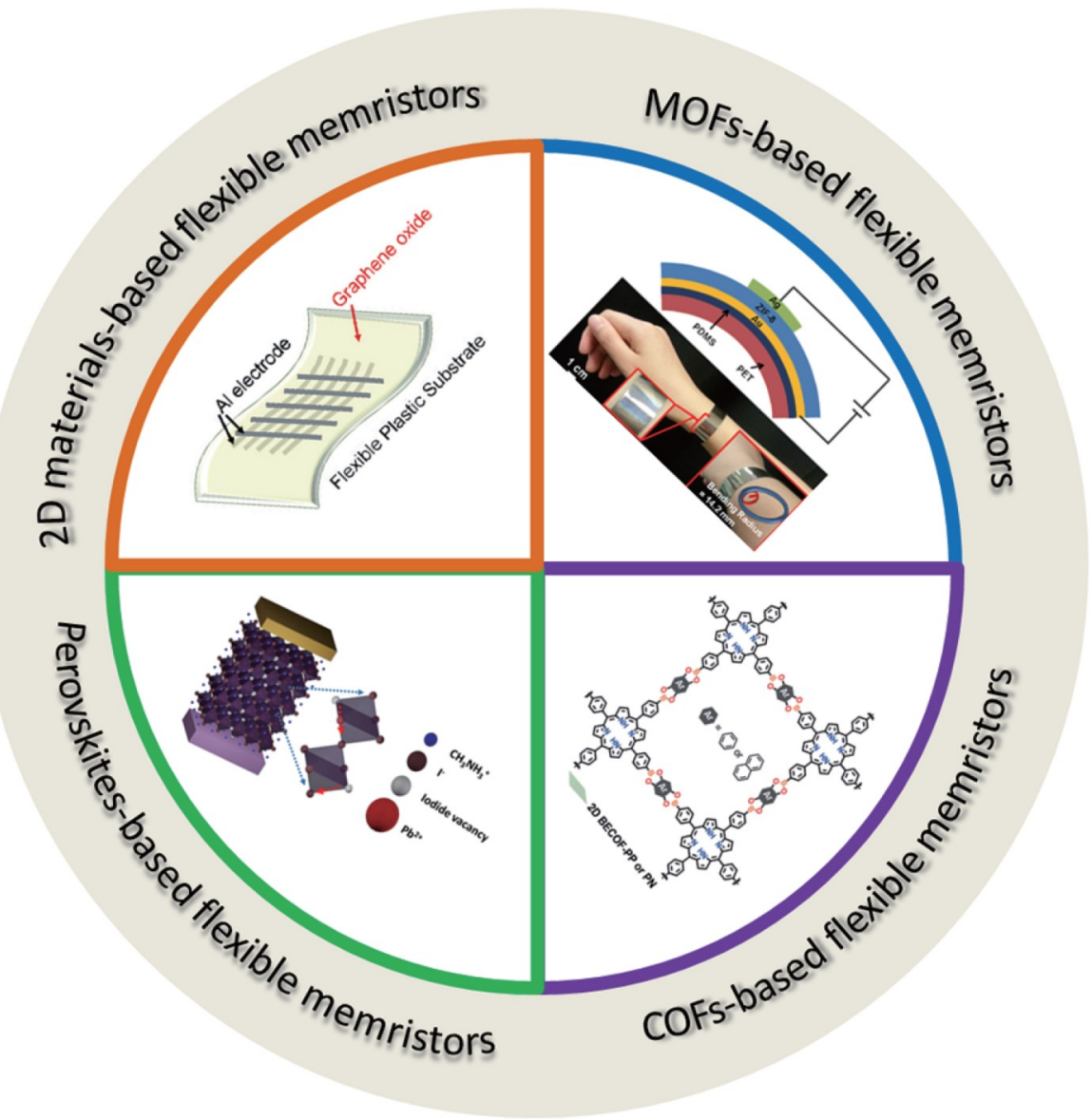

Figure 2 Schematic illustrations of various flexible CMs for memristor applications. 2D materials-based flexible memristors. Reprinted with permission from Ref. [45], Copyright 2010, American Chemical Society. MOFs-based flexible memristors. Reprinted with permission from Ref. [46], Copyright 2016, Wiley-VCH. COFs-based flexible memristors. Reprinted with permission from Ref. [47], Copyright 2020, Wiley-VCH. Perovskites-based flexible memristors. Reprinted with permission from Ref. [48], Copyright 2016, American Chemical Society. 
affording an efficient way of realizing diverse memory functions.

Lateral memristor device structure

In addition to the vertical structure, the lateral structure is also adopted for fabricating CMs-based flexible memristors, as illustrated by an example from $\mathrm{Vu}$ et al. (Fig. 3b) [50]. A lateral structure contains two transversely distributed electrodes and an active material channel between the electrodes. With the configuration of two laterally distributed electrodes, the memristive devices can be modulated via the gate voltage in the field-effect transistor (FET) geometry, which provides more opportunities for tuning the device performance. Taking the different functionalities into consideration, it is suggested to select an appropriate device structure in terms of the intrinsic nature and fabrication techniques of the memristive materials.

\section{Memristive switching mechanisms}

For memristors, it is important to understand the underlying memristive mechanisms of the switching characteristics. Over the past few years, several memristive mechanisms have been proposed for the CMs-based flexible memristors [52-55]. Here, we briefly summarize two types of mechanisms that are commonly utilized for clarifying the origin of most memristive behaviors, namely, ion migration and charge trapping/detrapping.

Ion migration

On the basis of theoretical and experimental analysis, ion migration has been recognized as a reasonable mechanism in a variety of memristors, which is closely correlated with the active materials and electrodes used in the devices [38,56,57]. Mobile ions (cations and/or anions) can migrate through the active material with the assistance of external electric field. For instance, by applying an electric voltage to the electrode of silver (Ag), $\mathrm{Ag}^{+}$ions can be formed through oxidation from the active electrode and injected into the resistive switching layer, which are then reduced at the counter electrode and aggregate into metal filaments (Fig. 4a) [58]. When the metal filaments eventually connect both electrodes, the conductivity of memristor will improve greatly, leading to LRS. By applying an opposite voltage bias, the metal filaments will decompose under the influence of electrochemical oxidization and switch the device from LRS to initial HRS. This mechanism corresponds to electrochemical metallization memory (ECM), which invokes a series of conductive bridging resistance random access memory (CB-RRAM) devices.

For CB-RRAM, an electroforming process is often required to generate conduction filaments based on the migration of metal ions or particles, which is typically conducted by exerting a high voltage/current to the resistive switching device [56,57]. Nevertheless, the application of high voltage is not desirable for the low power consumption. On the other hand, the electroforming process generally produces localized conduction filaments, resulting in the discrete and abrupt resistance transition that is incongruous for mimicking synaptic behavior. To this regard, Jo et al. [59] fabricated a memristor on the basis of a co-sputtered a

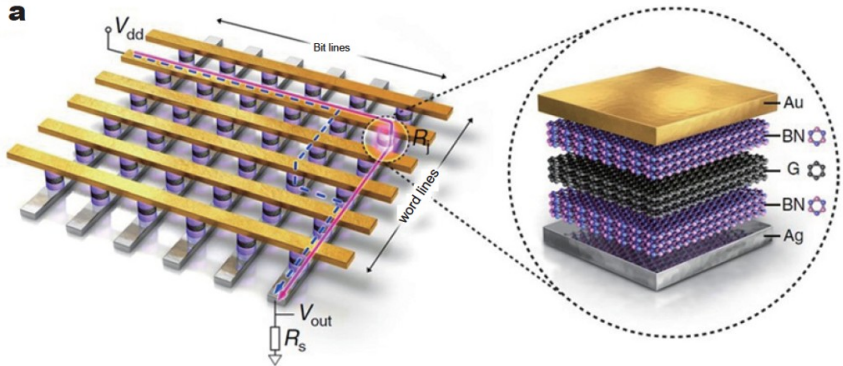

b

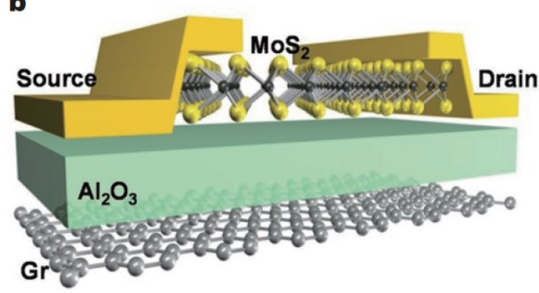

Figure 3 Schematic illustrations of two typical memristor device configurations. (a) Vertical MIM structure. Reprinted with permission from Ref. [51], Copyright 2019, under the Creative Commons License. (b) Lateral FET structure. Reprinted with permission from Ref. [50], Copyright 2017, Wiley-VCH.

a

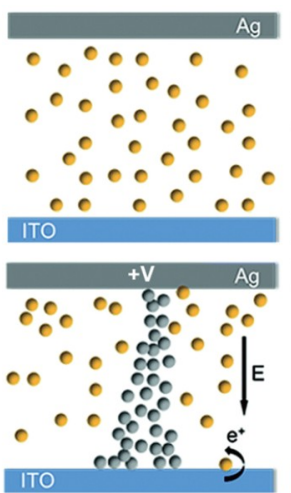

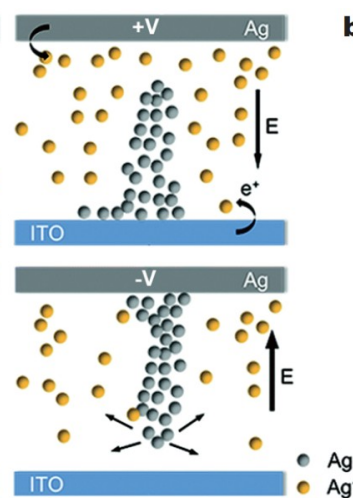

b I. Initial state

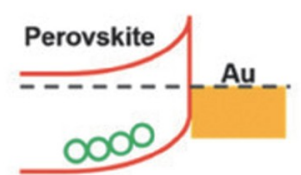

III. Remove electricity

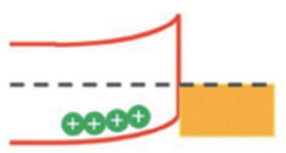

\section{SET process (1.5 V)}

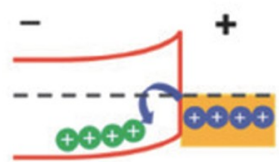

IV. RESET process

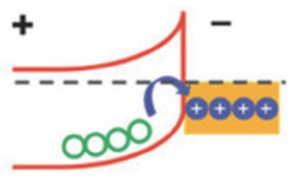

Figure 4 Schematic illustrations of different memristive mechanisms. (a) The formation and disruption of Ag metal filament on the basis of ion migration. Reprinted with permission from Ref. [58], Copyright 2020, The Royal Society of Chemistry. (b) Charge trapping/detrapping mechanism of the perovskitebased memristor. Reprinted with permission from Ref. [63], Copyright 2018, Wiley-VCH. 
$\mathrm{Ag}$ and $\mathrm{Si}$ active layer with a properly designed $\mathrm{Ag} / \mathrm{Si}$ gradient mixture ratio that facilitated the formation of an Ag-rich (high conductivity) region and an Ag-poor (low conductivity) region, which succeeded in eliminating the electroforming process, and hence led to reliable analog switching behaviors for neuromorphic application.

Valence change memory (VCM) is another type of filamentary-type conduction modes [60]. VCM relies on the anion migration and redistribution to form conductive filaments with a valence change, thereby altering the local conductivity of active layer. Taking the metal oxide-based memristor as an example, oxygen ions $\left(\mathrm{O}^{2-}\right)$ can migrate among the switching layer under an external electric field. The occurrence of oxygen vacancies will form oxygen-deficient filaments in the active layer, leading to oxygen-vacancy-based conductive switching. Under reverse voltage bias, oxygen vacancies combine with oxygen ions, resulting in the rupture of oxygen-deficient filaments and hence the recovery of HRS. Another example was proposed by Xu et al. [61], which demonstrated the interfacial effect on the ion migration of organometal halide perovskite (HP) $\left(\mathrm{CH}_{3} \mathrm{NH}_{3-}\right.$ $\mathrm{PbBr}_{3}$ ) for artificial synapse. A memristor of buffer-capped conducting polymer (BCCP)/ $/ \mathrm{CH}_{3} \mathrm{NH}_{3} \mathrm{PbBr}_{3} / \mathrm{Al}$ was fabricated, where the inserted BCCP layer could serve as a reservoir to trap mobile ions in perovskite. It was found that the crystallite formation of $\mathrm{CH}_{3} \mathrm{NH}_{3} \mathrm{PbBr}_{3}$ tended to be uniform due to the obvious interfacial effect caused by the BCCP layer. Moreover, the conductance of $\mathrm{CH}_{3} \mathrm{NH}_{3} \mathrm{PbBr}_{3}$ could be temporarily or persistently tuned by pulse-induced ion redistribution across the $\mathrm{CH}_{3} \mathrm{NH}_{3} \mathrm{PbBr}_{3}$ film or ion injection into the BCCP layer. When a strong pulse was applied, a fraction of ions migrated far enough to be trapped at the $\mathrm{CH}_{3} \mathrm{NH}_{3} \mathrm{PbBr}_{3} / \mathrm{BCCP}$ interface or even be injected into the BCCP to leave more vacancies. Accordingly, some of the halide sites in the $\mathrm{CH}_{3} \mathrm{NH}_{3} \mathrm{PbBr}_{3}$ became vacant to form conductive filaments, causing the memristive switching behaviors.

\section{Charge trapping/detrapping}

The charge trapping/detrapping due to the interfaces or active materials has been proposed as another effective mechanism in CMs-based resistive memories [30,41,62]. Taking the perovskitebased memristor as an example, the interface trap states have been claimed to be the probable causes of resistive switching behaviors. When using Au as an electrode, the Schottky barrier can form at the Au/perovskite interface due to the Fermi level difference between the electrode and the active material (Fig. 4b) [63]. By applying an electric field to the gold $(\mathrm{Au})$ electrode, the Schottky barrier could be reduced, which facilitates the hole injection from the electrode to the interfacial trapping centers. The contact between the Au electrode and perovskite turns to be quasi-ohmic because the Fermi level of perovskite shifts to the valence band, leading to the transition from HRS to LRS. Moreover, by exerting a reverse voltage bias to the Au electrode, the trapped holes will release from the interface trapped states. The Schottky barrier increases and recovers to the initial state, making the memristor switch back to the initial HRS.

\section{D MATERIALS-BASED FLEXIBLE MEMRISTORS}

2D materials have been regarded as a class of promising candidates for next-generation electronic devices, neuromorphic electronics and artificial sensory systems, due to their appealing physical, chemical and electronic features [26,64-69]. 2D materials possess a lot of unique characteristics such as ultrathin thickness, large specific surface area, excellent mechanical flexibility, and high transparency, which endow them with potential of implementing high-performance flexible and wearable electronics. In particular, the memristive effects have been observed in various $2 \mathrm{D}$ materials in the past few years, such as graphene oxide (GO), transition metal dichalcogenides (TMDs, such as $\mathrm{MoS}_{2}$ ), black phosphorus (BP), boron nitride (BN), and MXenes [8,23,56,70-72].

As the early member of $2 \mathrm{D}$ materials family, $\mathrm{GO}$ and its derivatives (e.g., polymer-functionalized $\mathrm{GO}, \mathrm{TiO}_{2}$-assisted $\mathrm{GO}$, and $\mathrm{GO} /$ biomaterial composites) have been intensively investigated for fabricating flexible devices, which succeed in realizing the reliable memristive switching behaviors [65,73-75]. For instance, Jeong et al. [45] reported the fabrication of a flexible memristor by utilizing GO as the active layer (Fig. $5 \mathrm{a}$ ). In this flexible device, crossbar arrays of aluminum $(\mathrm{Al}) / \mathrm{GO} / \mathrm{Al}$ cells on the flexible polyethersulfone foil were acquired. The resultant device exhibited typical bipolar reversible memory characteristics with an ON/OFF current ratio of $~ 100$ (Fig. 5b), and robust device performance over 1000 bending cycles (Fig. 5c). It is believed that the switching behavior of the device originated from a dual mechanism of oxygen migration and $\mathrm{Al}$ diffusion under the electric field. Notably, Zhao et al. [74] adopted an efficient $\mathrm{TiO}_{2}$-assisted photocatalytic reduction method to locally manipulate the size and fraction of reduced GO (rGO)domains, which successfully eliminated the electrical forming process during the device operation. The elimination of the electrical forming process facilitated to suppress the overgrowth of rGO conducting filaments and hence improve resistive memory characteristics of $\mathrm{GO}-\mathrm{TiO}_{2}$ nanocomposites. As a result, the as-fabricated flexible memristor on indium tin oxide (ITO)-coated polyethylene terephthalate (PET) substrate exhibited excellent mechanical flexibility (Fig. 5d). The resistive switching curves before and after 10,000 bending cycles showed no degradation (Fig. 5e), including SET/RESET voltage and current levels of ON/OFF states (Fig. 5f). It is worth mentioning that the highly reduced graphene can also serve as non-metal electrodes for flexible electronics. Wang et al. [8] reported a robust memristor based on fully layered 2D materials using graphene as electrodes. The as-prepared vertical multi-layered device of graphene/ $\mathrm{MoS}_{2-x} \mathrm{O}_{x} /$ graphene exhibited repeatable bipolar resistive switching even at the temperature of $340^{\circ} \mathrm{C}$. Through the in situ scanning transmission electron microscopy (TEM) analysis, the migration of oxygen ions was confirmed to be the origin for the memristive properties. Moreover, they fabricated graphene/ $\mathrm{MoS}_{2-x} \mathrm{O}_{x} /$ graphene on the polyimide substrate and found that the flexible memristor presented excellent mechanical flexibility over 1000 bending times, indicating potential flexible electronic applications.

Particularly, in the development of 2D graphene-based flexible electronics, there is an increasing interest in fabricating neuromorphic devices, which holds great promise for artificial intelligent computing hardware [76]. As a typical representation, Xiong et al. [65] demonstrated a flexible cognitive memristor based on poly(vinyl alcohol)-GO nanocomposites (PVA-GO), which could realize Pavlovian-associative learning behaviors. Fig. $6 \mathrm{a}$ shows the schematic illustration of neural networks in Pavlovian conditioned reflex. The "food" signal (US: unconditioned stimulus) could make the dog salivate (UR: unconditioned response). However, the "bell" signal (NS: neutral 

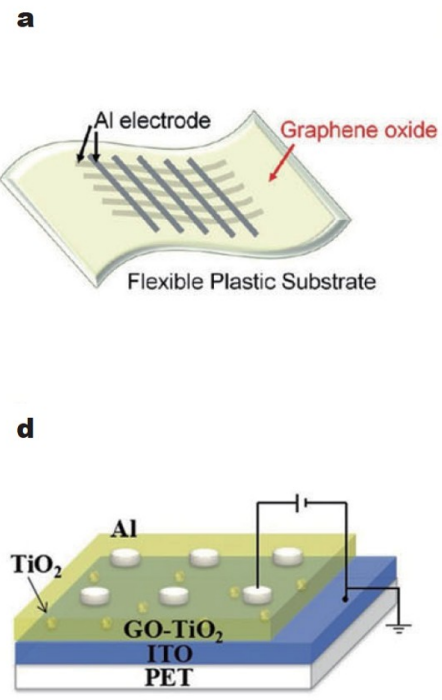
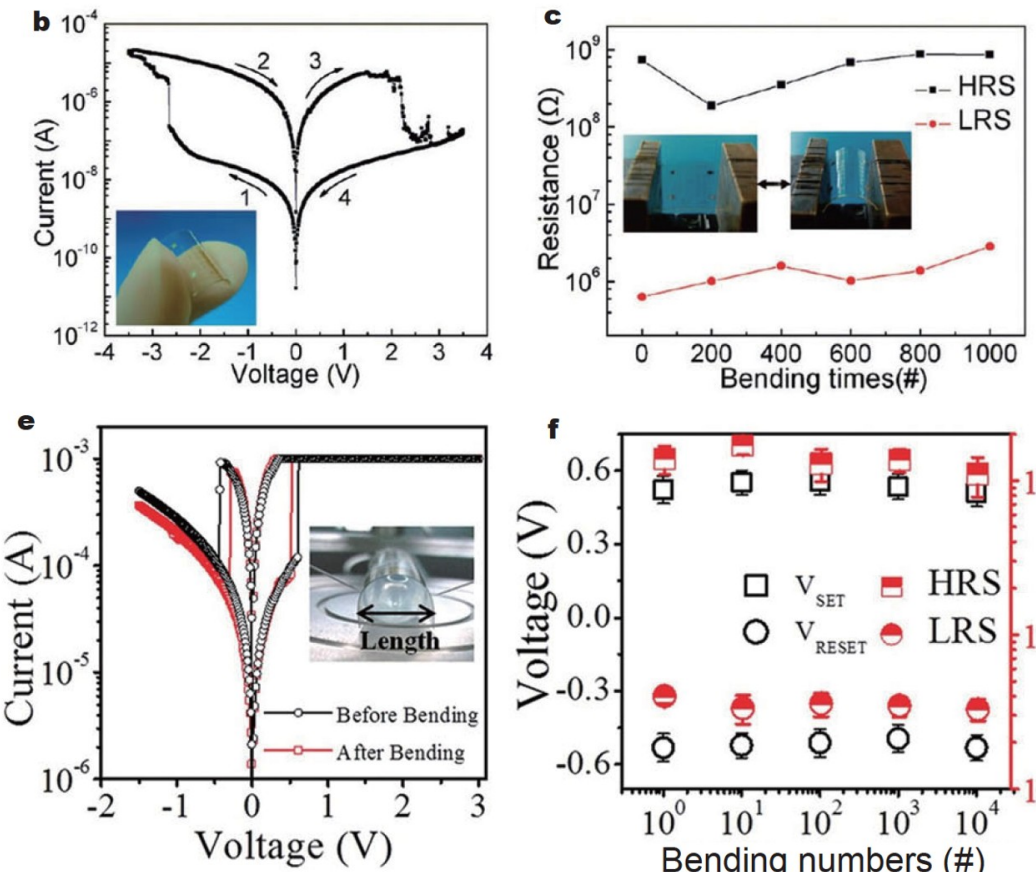

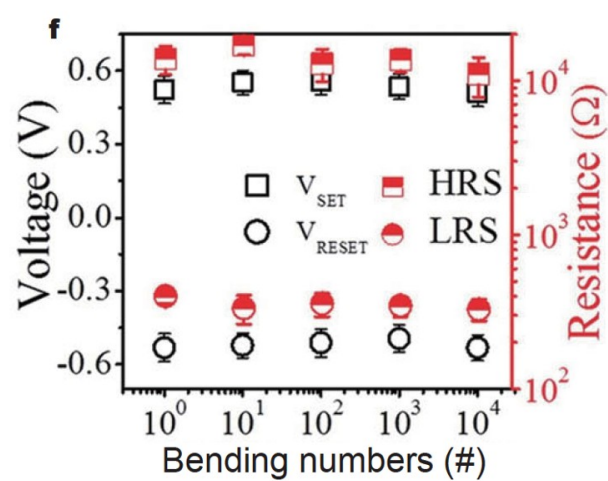

Figure 5 (a) A schematic illustration of the flexible $\mathrm{Al} / \mathrm{GO} / \mathrm{Al}$ crossbar memristor. (b) Typical current-voltage (I-V) curve of the flexible Al/GO/Al memristor. (c) Continuous bending test of the flexible Al/GO/Al memristor. Reprinted with permission from Ref. [45], Copyright 2010, American Chemical Society. (d) A schematic illustration of the flexible Al/GO- $\mathrm{TiO}_{2} / \mathrm{ITO}$ memristor. (e) Typical $I$ - $V$ curves of the flexible Al/GO-TiO $/$ ITO memristor before and after bending. (f) Endurance performance of the flexible $\mathrm{Al} / \mathrm{GO}-\mathrm{TiO}_{2} / \mathrm{ITO}$ memristor over $10^{4}$ continuous bending cycles. Reprinted with permission from Ref. [74], Copyright 2018, Wiley-VCH.

stimulus) could not initially induce salivation. When both "food" signal and "bell" signal simultaneously happen, the dog will salivate. Thus, a training behavior is triggered and an association between "bell" and "food" is eventually established. After training, the dog will salivate even "bell" signal is merely offered. In such process, "salivation" is regarded as conditional response (CR), and the NS is regarded as conditional stimulus (CS). Fig. 6b shows the four typical RS's of the as-fabricated flexible PVA-GO-based memristor, namely as LWS1, LWS2, HWS1, and HWS2 (LWS: low synaptic weight state; HWS: high synaptic weight state). As shown in Fig. 6c, the authors designed the memristor-based neuron circuit to mimic the Pavlovian conditional reflex. Three memristors of M1, M2, and M3 were integrated. M1 and M2 stayed in LRS and HRS, respectively. Accordingly, the M3 will output the "salivation" response. The initial weight of M1 was set to HWS1, while that of the initial weight of M2 was set to LWS2. The "food" stimulus and "bell" stimulus could be applied to M1 and M2, respectively. The interval time between the "food" and "bell" signals was set to be $20 \mathrm{~ms}$ (Fig. 6d), which established the association between "bell" and "food". Fig. 6e-h display the training results. It can be noted that after the training, the "bell" stimulus (CS) alone would succeed in triggering strong response in axon membrane current, indicating the "salivation" of CR (Fig. 6h). Thus, the three memristors-based neuron circuits can realize the Pavlovian learning behavior, which are promising for fabricating multifunctional perception learning systems.

BP-based CMs have also aroused numerous research interests owing to their unique structures and outstanding optoelectronic properties. Recently, they have shown potentials in flexible memristors [71,77,78]. In 2015, Zhang et al. [72] reported a facile solution-based method for the preparation of BP quantum dots (BPQDs), which owned an average size of $4.9 \pm 1.6 \mathrm{~nm}$ and thickness of $1.9 \pm 0.9 \mathrm{~nm}$ (about $4 \pm 2$ layers) (Fig. 7a). They dispersed BPQDs into polyvinylpyrrolidone (PVP) and successfully fabricated a flexible memristor with an MIM structure of Ag/BPQD-PVP/Au on PET substrate (Fig. 7b). The flexible device based on these uniformly sized BPQDs (Fig. 7c) exhibited nonvolatile rewritable resistive memory behavior with a high $\mathrm{ON} / \mathrm{OFF}$ current ratio over $6.0 \times 10^{4}$ and long retention time of $1.1 \times 10^{3} \mathrm{~s}$ (Fig. 7d, e). Tian et al. [77] recently reported a BPbased ambipolar memristor with a lateral structure, which showed dynamically reconfigurable and polarity reversible switching behavior. Besides, the BP-based memristor displayed four different memory states with high electron mobility. It was discovered that the ambipolar conduction in BP channel ensured the tunable programmed/erased states, which offer adaptable properties for many emerging applications such as artificial synapses that can dynamically reconfigure between excitatory and inhibitory responses.

Intriguingly, the combination of $2 \mathrm{D}$ materials can implement large-scale crossbar arrays for energy-efficient 3D memory and neuromorphic computing hardware systems [8,23,51,78]. For example, Sun et al. [51] demonstrated a novel memory cell based on a van der Waals heterostructure of $2 \mathrm{D}$ hexagonal $\mathrm{BN}$ (h-BN) and graphene (Fig. 3a). Through vertically stacking h-BN and graphene layers into a $12 \times 12$ crossbar arrayed structure of $\mathrm{Au} /$ h-BN/graphene/h-BN/Ag (Fig. 8a), nonvolatile and volatile memory operations were both obtained within the two h-BN layers, which afforded a self-selectivity of $1.0 \times 10^{10}$ with a high ON/OFF ratio over 1000 . The insertion of graphene layer between the two h-BN layers effectively blocked the diffusion of 

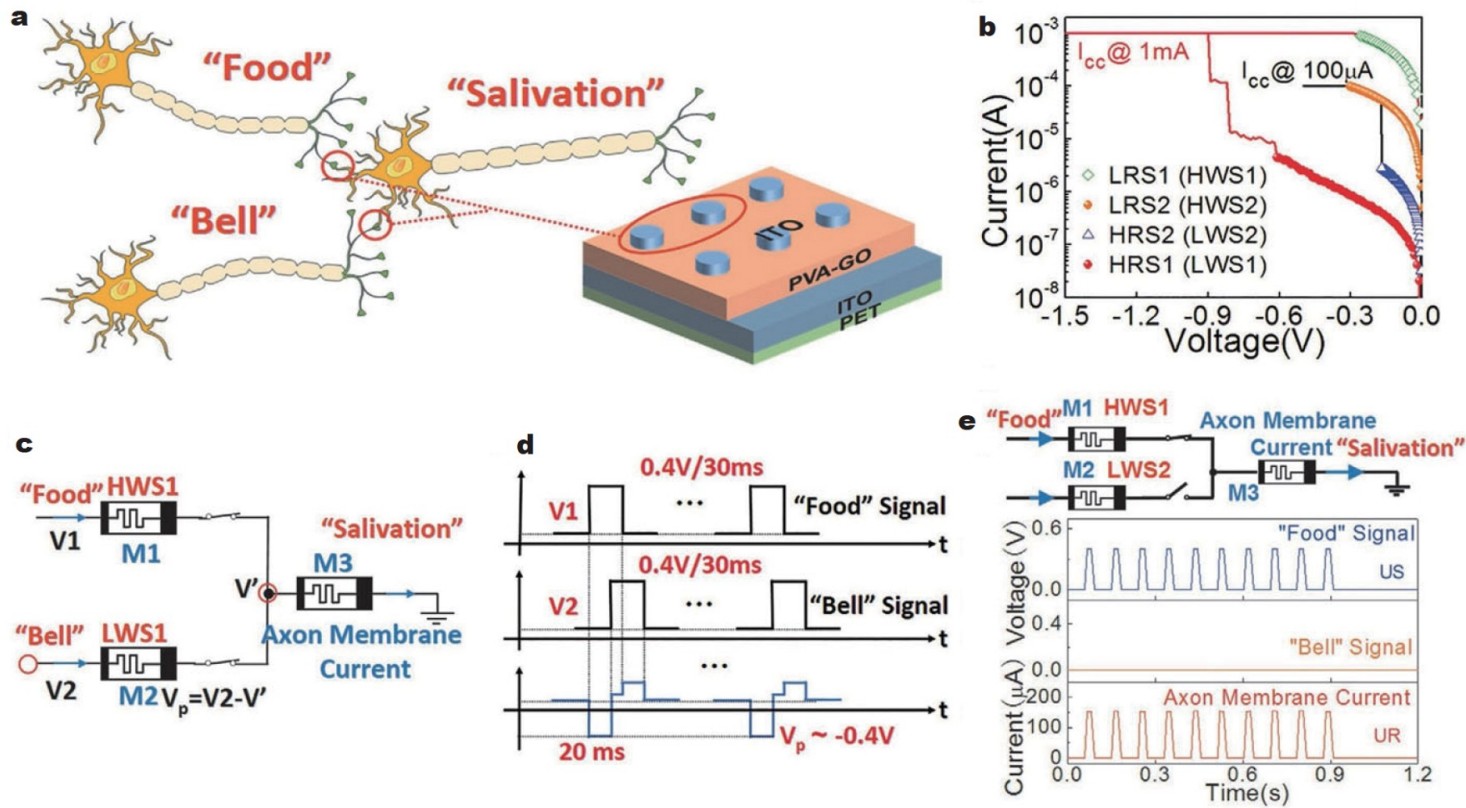

f
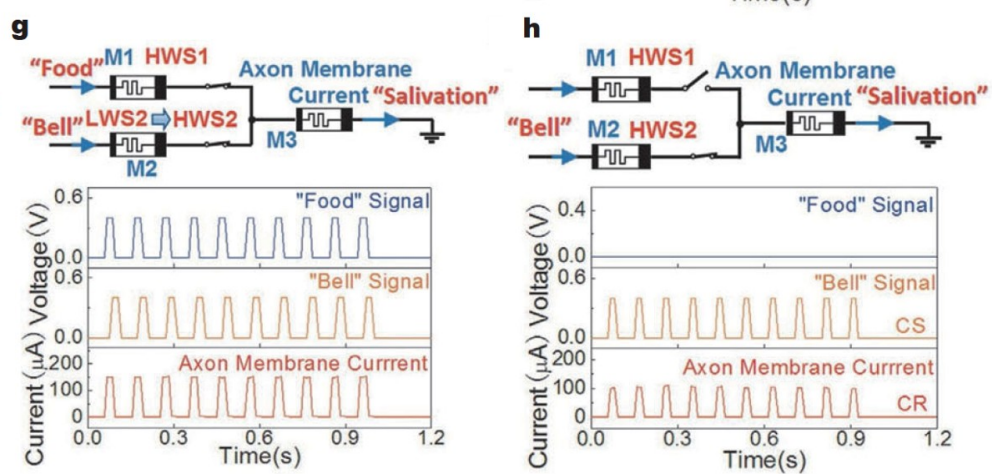

Figure 6 (a) Schematic diagram of neural networks in Pavlovian conditioned reflex. (b) $I-V$ curves for four RS's of the device, including LWS1, LWS2, HWS1, and HWS2. (c) Memristors-based neuron circuit to realize the Pavlovian conditioned reflex. (d) Schematic diagram for the training process of Pavlovian associative learning behaviors. Ten pairs of "food" and "bell" stimuli are applied with intervals of 20 ms. (e) "Food" signal induces strong axon membrane current responses. (f) "Bell" signal induces no axon membrane current response. (g) Associative learning process: paired "Food" and "Bell" stimuli induce strong axon membrane current responses. M2 is switched from LWS2 to HWS2 in this process. (h) "Bell" signal after association induces strong axon membrane current responses. Reprinted with permission from Ref. [65], Copyright 2020, Wiley-VCH.

Ag filaments, which helped to integrate the volatile and nonvolatile kinetics of $\mathrm{h}-\mathrm{BN}$ in an efficient way. Moreover, they repeated the self-selective memory operations of this memristive device on a flexible PET substrate (Fig. 8b-d), which allowed for future memory applications such as the wearable artificial intelligent devices. In the same year, $\mathrm{Wu}$ et al. [79] used monolayer h-BN as the insulator in an atomic memristor. With the assistance of the extremely thin one-atom-layer h-BN sheet (about $0.33 \mathrm{~nm}$ ), not only forming-free switching and high ON/ OFF ratio $\left(1.0 \times 10^{7}\right)$ were attained in unipolar and bipolar operations, but also a record vertical scaling limit of memory materials was successfully broken. The molecular simulations attributed the memristive performance to the incorporation of metal ions into h-BN vacancies under the voltage bias. Such hBN-based memory materials hold huge potential to construct flexible devices for logic, data-adaptive memory, and neuromorphic computing applications.

Among 2D materials, MXenes have recently emerged as outstanding candidates for the application of catalysis, energy storage, sensors, and memristive electronics [80-84]. MXenes with the general formula of $\mathrm{M}_{n+1} \mathrm{X}_{n} \mathrm{~T}_{x}(n=1-4)$ are commonly synthesized from "MAX" by removing A layer, where $\mathrm{M}$ represents a transition metal, A represents an element from group 13 or 14 in the periodic table of elements, $\mathrm{X}$ represents carbon and/or nitrogen, and $\mathrm{T}_{x}$ represents various surface termination. So far, over thirty different MXenes have been reported. Besides, the surface modification engineering of MXenes has also attracted a lot of research attention [85]. Sun et al. [86] recently reported the octylphosphonic acid-modified MXene of $\mathrm{Ti}_{3} \mathrm{C}_{2} \mathrm{~T}_{x}$ and utilized it as the dielectric layer for flexible multilevel memory device (Fig. 9a). The stable ternary memory behavior (yield 58\%) was obtained from the MXene-based device, which was explained by the mechanism of charge trapping and filamentary conduction. The flexible MXene-based device showed stable ternary performance without degradation after $4000 \mathrm{~s}$, or even after bending over 5000 cycles under a curvature radius of $2.1 \mathrm{~cm}$ (Fig. 9b). Furthermore, MXenes also own the potential for neuromorphic computing applications. Khot et al. [70] investigated the bipolar resistive switching and synaptic learning properties of the $\mathrm{Ti}_{3} \mathrm{C}_{2}$ MXene-based memristors (Fig. 9c). By 

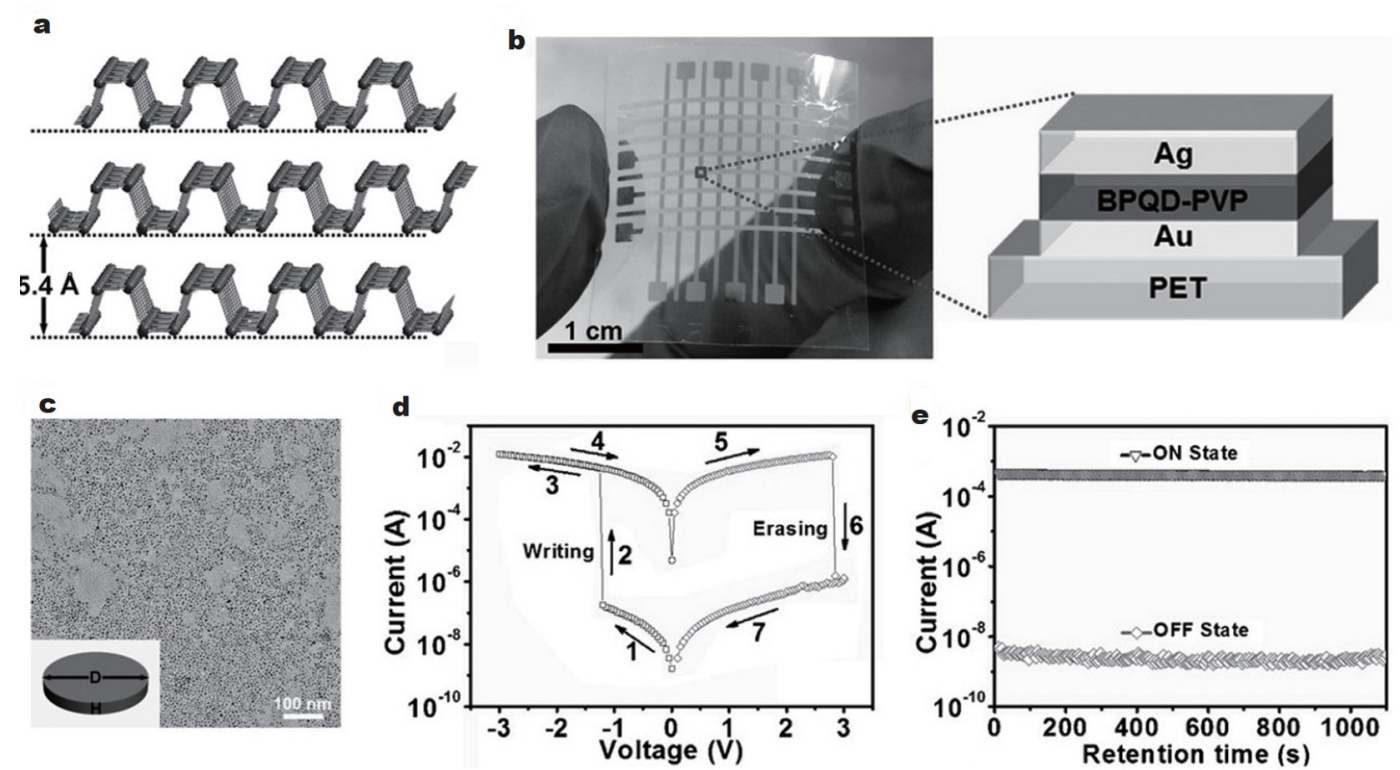

Figure 7 (a) Schematic diagram of BP crystal. (b) Photograph and illustration of the fabricated flexible Ag/BPQD-PVP/Au memristor. (c) TEM image of the as-prepared BPQDs. (d) $I-V$ characteristics of the flexible Ag/BPQD-PVP/Au memristor. (e) Retention test of the flexible Ag/BPQD-PVP/Au memristor. Reprinted with permission from Ref. [72], Copyright 2015, Wiley-VCH.

a

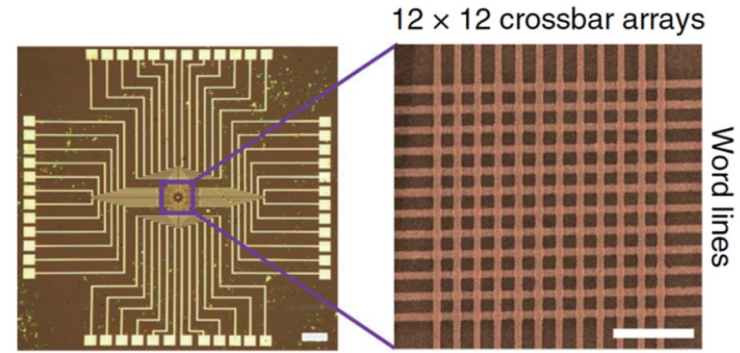

Bit lines
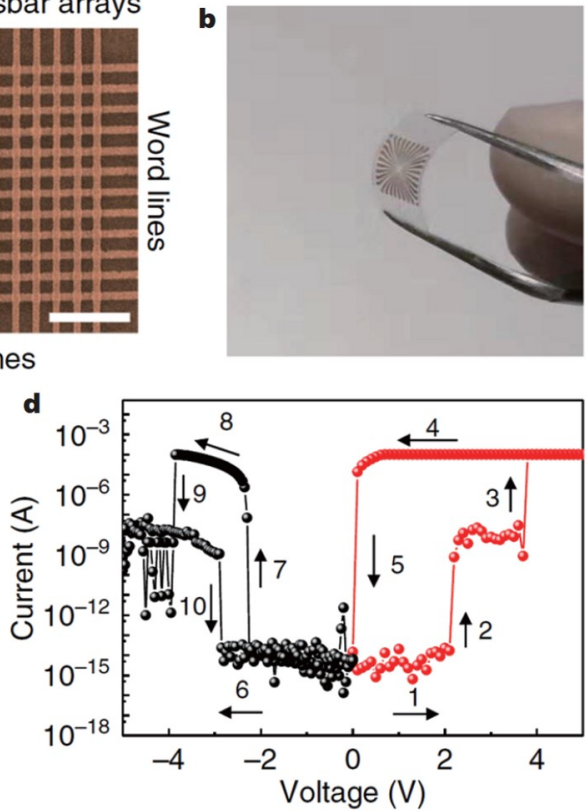

Figure 8 (a) Optical and scanning electron microscopy (SEM) images of the Ag/h-BN/graphene/h-BN/Au memristor in the $12 \times 12$ crossbar memory array architecture. Scale bars: 200 and $5 \mu \mathrm{m}$ for the optical and SEM images, respectively. (b, c) Optical images of the flexible Au/h-BN/graphene/h-BN/Ag memristor on PET substrate. (d) A full-voltage-range resistive switching curve of the flexible Au/h-BN/graphene/h-BN/Ag memristor. Reprinted with permission from Ref. [51], Copyright 2019, under the Creative Commons License.

altering the top electrodes ( $\mathrm{Ag}$, platinum $(\mathrm{Pt})$, and $\mathrm{Al})$, the optimal memristive performance was obtained from the $\mathrm{Al} /$ $\mathrm{Ti}_{3}-\mathrm{C}_{2} / \mathrm{Pt}$ device, which could be ascribed to the excellent electron transport under the mechanism of filamentary conduction. As depicted in Fig. 9d, e, the potentiation/depression synaptic properties and the spike-timing-dependent plasticity (STDP)based Hebbian learning rules could be mimicked by the memristive device. These above-mentioned studies demonstrate that 2D CMs possess satisfactory compatibility with the flexible substrates and therefore can be considered as potential candidates for future flexible nonvolatile memory and synaptic learning applications.

\section{MOFs-BASED FLEXIBLE MEMRISTORS}

MOFs are categorized as porous CMs constructed by metal centers and organic linkers [87,88]. On the basis of their versatility, MOFs that possess different pore sizes and crystal structures have been utilized in the fields of separation, catalysis, 

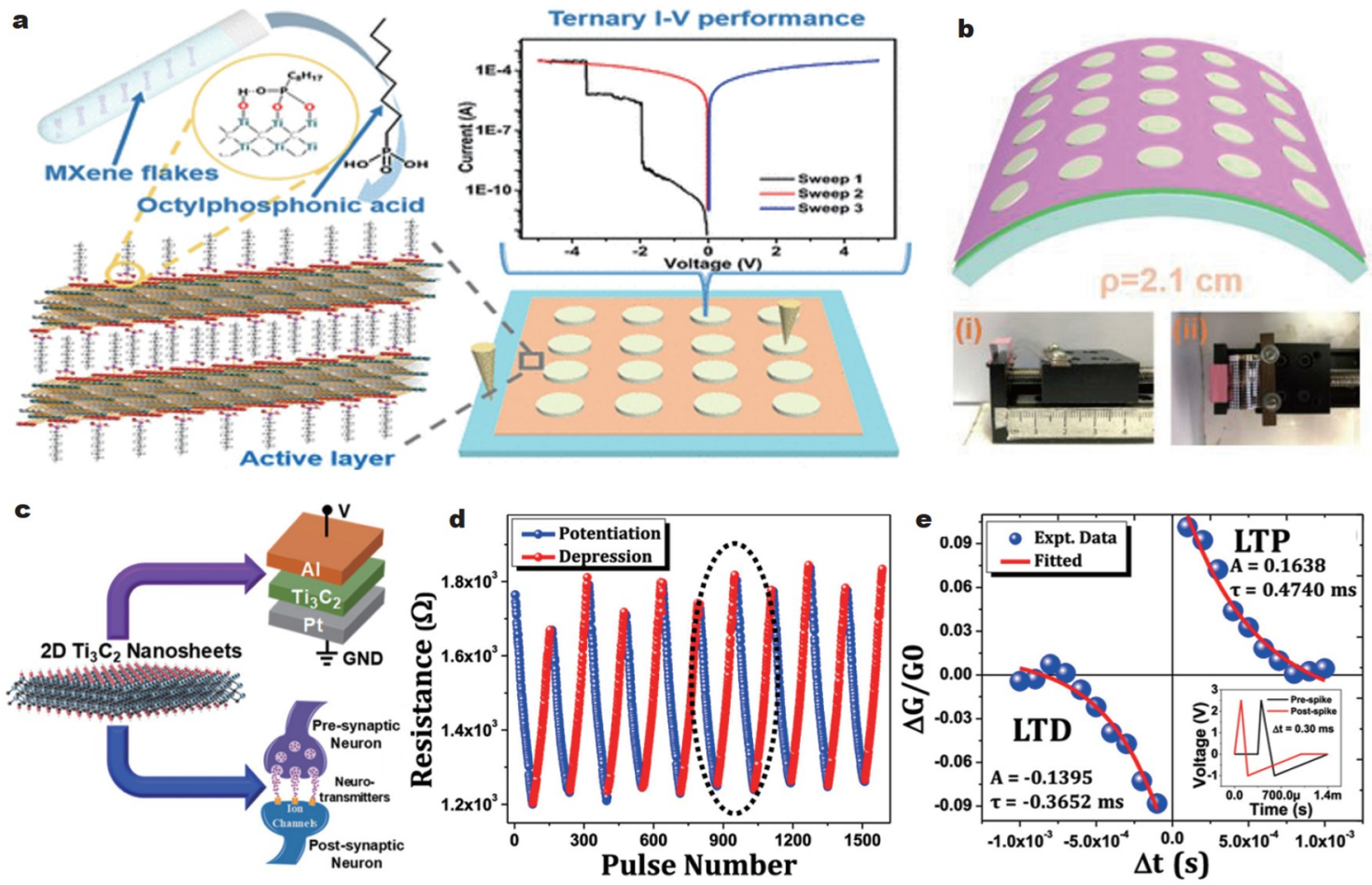

Figure 9 (a) Schematic diagram of the octylphosphonic acid-modified $\mathrm{Ti}_{3} \mathrm{C}_{2} \mathrm{~T}_{x}$ MXene and the ternary $I-V$ characteristics of the Ti ${ }_{3} \mathrm{C}_{2} \mathrm{~T}_{x} \mathrm{MXene}$-based flexible memory device. (b) Schematic diagram of the $\mathrm{Ti}_{3} \mathrm{C}_{2} \mathrm{~T}_{x}$ MXene-based flexible memory device. Reprinted with permission from Ref. [86], Copyright 2020, American Chemical Society. (c) Schematic diagram of the $2 \mathrm{D} \mathrm{Ti}_{3} \mathrm{C}_{2}$ MXene-based memristor. (d) Potentiation and depression synaptic properties of the 2D $\mathrm{Ti}_{3} \mathrm{C}_{2}$ MXene-based memristor. (e) STDP-based anti-symmetric Hebbian learning rules mimicked using the $\mathrm{Al} / \mathrm{Ti}_{3} \mathrm{C}_{2} / \mathrm{Pt}$ memristor. Reprinted with permission from Ref. [70], Copyright 2021, American Chemical Society.

chemical sensors, and energy storage [89-92]. Moreover, in the process of continuous research, MOFs have been reported as promising candidates for flexible resistive switching and memristor applications [28,46,93-100].

In 2015, Pan et al. [98] reported a high-quality MOF nanofilm of HKUST-1 (i.e., $\mathrm{Cu}_{3}(\mathrm{BTC})_{2}$, BTC = benzene-1,3,5-tricarboxylic acid) on flexible PET substrate. The obtained $\mathrm{Au} /$ HKUST-1/Au flexible device afforded uniform and reproducible memristive switching characteristics (Fig. 10a), which could be maintained even under the high strain level (Fig. 10b) and more than $1.0 \times 10^{7}$ endurable cycles (Fig. 10c). Moreover, through the cooperation of conductive atomic force microscopy and depthprofiling X-ray photoelectron spectroscopy analyses, they proposed that the electric field-induced migration of the $\mathrm{Cu}^{2+}$ ions was responsible for the resistance switching in HKUST-1 nanofilms. The same group also fabricated an intrinsically stretchable memristor by utilizing GaInSn@poly(dimethyl siloxane) (PDMS) composites as soft electrode and MOF of MIL-53 as an insulator (Fig. 10d) [93]. They discovered that GaInSn@PDMS composites could display a high conductivity of $1.3 \times 10^{3} \mathrm{~S} \mathrm{~cm}^{-1}$ at strains over $80 \%$ and the fracture strain was as large as $108.14 \%$, which could function as an ideal soft electrode. The flexible Ag/MIL-53/GaInSn@PDMS memristor showed bipolar reproducible resistive switching characteristics even at the stretching strain of $10 \%$ (Fig. 10e) and more than 200 cycles (Fig. 10f). They further proposed that depositing single-crystalline MIL-53 nanofilm as the active layer and adopting silver nanowire-polymer nanocomposites as the stretchable electrode may extend the deformation capability of the flexible MOFbased devices.
As another example, Liu et al. [46] took advantages of a typical MOF material of zeolitic imidazolate framework-8 (ZIF-8) and prepared a flexible memristor with the structure of Ag/ZIF-8/ $\mathrm{Au} / \mathrm{PDMS} / \mathrm{PET}$ (Fig. 11a). The device succeeded in affording rewritable resistive switching behavior, which was ascribed to the formation of a conductive channel arisen from Ag nanoparticles among the ZIF-8 layer. Moreover, the conductivity of ZIF-8 could be modulated by adsorbing methanol molecules, which could thus achieve multilevel memory performance in the methanol vapor atmosphere (Fig. 11b). Furthermore, they demonstrated ZIF-8-based devices on soft substrates could well maintain the alcohol-mediated resistances under the bending state (Fig. 11c), which indicates the potential application of chemically mediated MOF-based devices for environmentresponsive wearable electronics. Similarly, Park et al. [96] also fabricated a flexible MOF memristor based on ZIF-8 thin film. The device of Al/ZIF-8/Au/PET demonstrated reproducible and reliable bipolar resistive switching characteristics with long retention time for up to $4000 \mathrm{~s}$ and satisfactory bending stability over 100 bending cycles.

Despite the utilization of single-component MOF-based CMs as shown above, it has been documented that embedding $2 \mathrm{D}$ nanomaterials into MOFs can further extend their potential versatility. For instance, Huang et al. [100] conducted the coating of various 2D materials including $\mathrm{MoS}_{2}, \mathrm{GO}$, and rGO nanosheets with ZIF-8 MOF material (e.g., MoS $@$ @IF-8 as shown in Fig. 11d). As a proof of concept, they further fabricated a flexible resistive memory device based on $\mathrm{MoS}_{2} @ Z I F-8$ on the PET substrate, which exhibited the nonvolatile memory effect with a high ON/OFF ratio over $7.0 \times 10^{4}$ (Fig. 11e) and long 

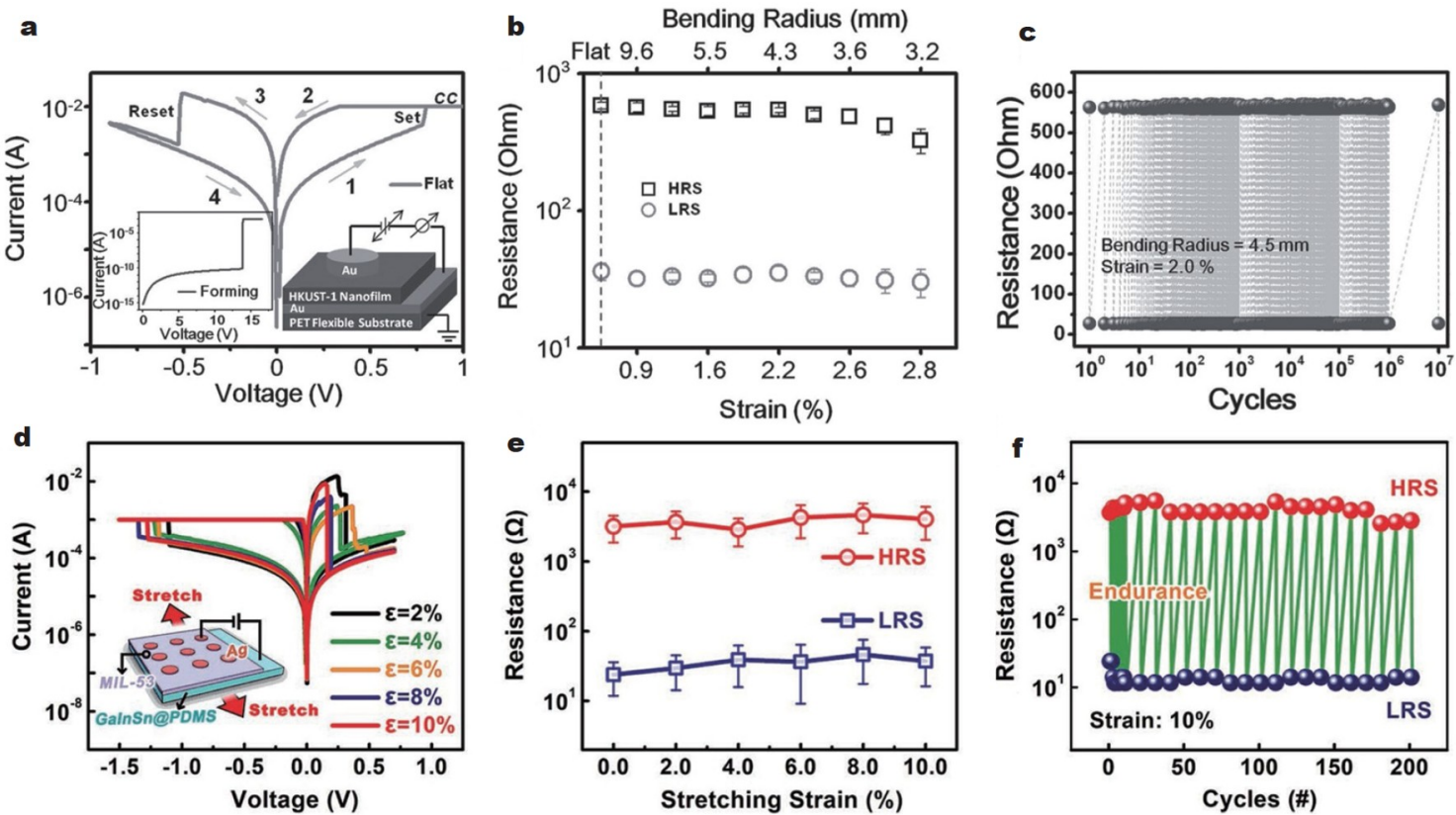

Figure 10 (a) $I-V$ characteristics of the flexible Au/HKUST-1/Au/PET memristor at the flat state. (b) HRS/LRS resistances of the flexible Au/HKUST-1/Au/ PET memristor as a function of the bending radius and strain level. (c) Endurance of HRS/LRS resistances at the strain level of $2.0 \%$. Reprinted with permission from Ref. [98], Copyright 2015, Wiley-VCH. (d) $I-V$ characteristics of the flexible Ag/MIL-53/GaInSn@PDMS memristor under the stretching strains of 2\%-10\%. (e) HRS/LRS resistances of the flexible Ag/MIL-53/GaInSn@PDMS memristor as a function of the strain level. (f) Endurance of HRS/LRS resistances at the stretching strain level of $10 \%$. Reprinted with permission from Ref. [93], Copyright 2018, Wiley-VCH.
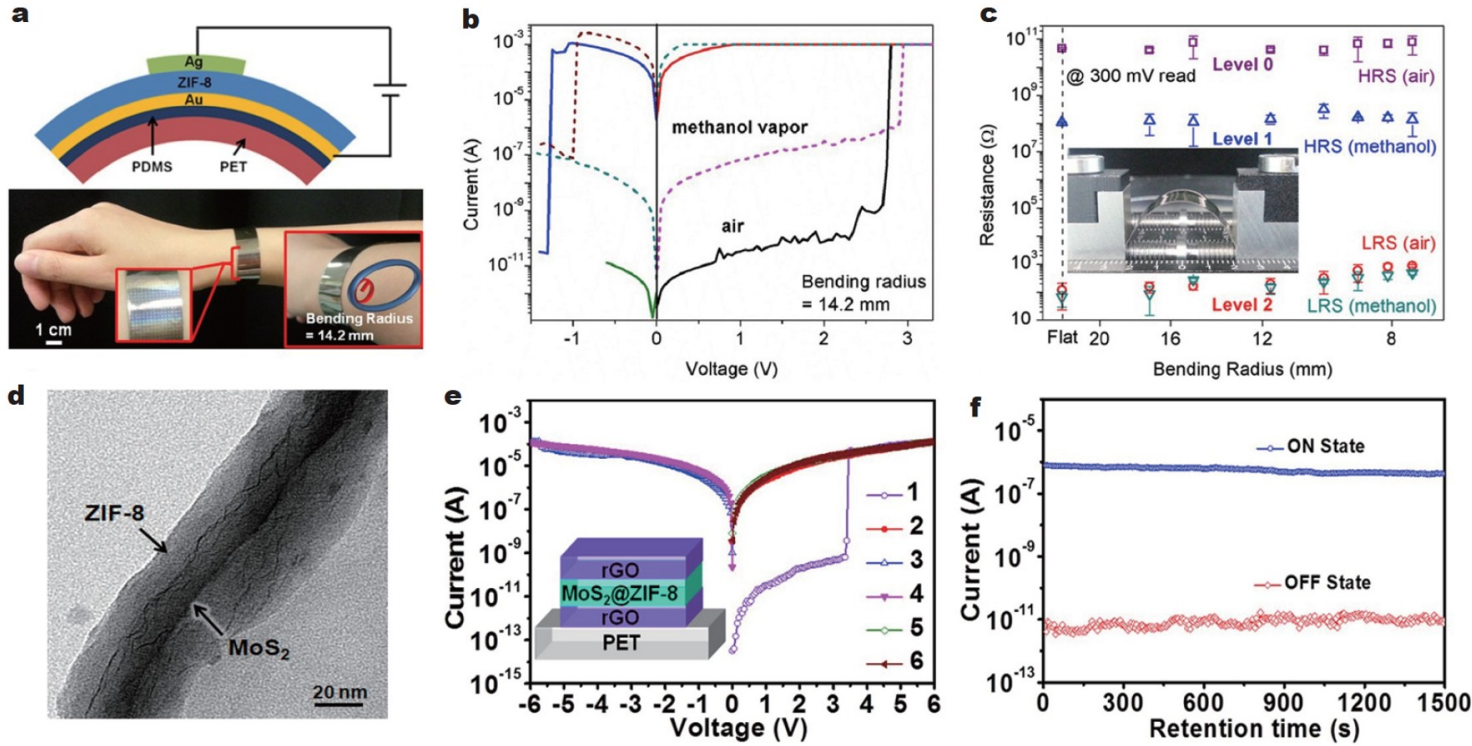

Figure 11 (a) Schematic structure of the flexible Ag/ZIF-8/Ag/PDMS/PET memristor and photograph of the device on a wrist strap with a minimal bending radius of $14.2 \mathrm{~mm}$. (b) $I-V$ curves of the flexible Ag/ZIF-8/Ag/PDMS/PET memristor in saturated methanol vapor and air at bending state. (c) RSs of the flexible Ag/ZIF-8/Ag/PDMS/PET memristor performed in air and methanol vapor versus the bending radius. Reprinted with permission from Ref. [46], Copyright 2016, Wiley-VCH. (d) TEM image of a curled $\mathrm{MoS}_{2} @ Z I F-8$ structure. (e) I-V curves of the flexible rGO/MoS @ZIF-8/rGO/PET memristor (f) Retention test of the flexible rGO/MoS ${ }_{2} @ Z I F-8 /$ rGO/PET memristor. Reprinted with permission from Ref. [100], Copyright 2014, American Chemical Society.

operating lifetime over $1.5 \times 10^{3} \mathrm{~s}$ (Fig. 11f). From these representative examples, it can be noted that MOF-based CMs are emerging as promising candidates for high-performance flexible memristor applications.

\section{COFs-BASED FLEXIBLE MEMRISTORS}

COFs are known as a class of organic porous CMs that possess the covalently connected networks and precisely stacked structures [101-103]. Thanks to their great designability, controllable topologies, oriented structures, and tunable functionalities, COFs have gained numerous interests in energy storage, catalysis, electrochemistry, and optoelectronics [104-107]. The electronic properties of COFs can be well modified through tailoring the structures of the linking units. Recently, a variety of 
COF-based materials have been developed for obtaining highly efficient organic semiconductors, such as FETs and photodetector devices. Moreover, the progress of thin-film processability and device integration has endowed COFs with extensive potential in resistive memory and memristor applications $[47,108,109]$.

Recently, one typical study was reported by Liu et al. [109], which afforded a wafer-scale ultrathin 2D imine polymer (2DPCOF) film at the air-water interface via a Schiff base condensation between benzene-1,3,5-tricarbaldehyde and $p$-phenylenediamine. Taking advantages of the porous structure of 2DPCOF polymer, they acquired a nonvolatile memristor based on the metal filamentary conductive mechanism. The as-fabricated $\mathrm{Ag} / 2 \mathrm{DP} / \mathrm{ITO}$ device showed bipolar resistive switching behavior with a high current ratio over $10^{5}$ (Fig. 12a), long retention time of $3.5 \times 10^{4} \mathrm{~s}$, and reliable endurance over 200 cycles (Fig. 12b). Additionally, the resistance of 2DP-based memristor could change in different organic solvents, which manifested the possibility of implementing multilevel data storage. Moreover, as the robust covalent linkages and amorphous nature provided 2DP with high flexibility and uniformity, they took advantages of the intrinsic flexibility of 2DP for flexible devices. The flexible memristor with a vertical $\mathrm{Ag} / 2 \mathrm{DP} /$ graphene structure exhibited reversible resistive switching behavior with excellent mechanical durability over 500 bending cycles (Fig. 12c, d).

Following the above study, Park et al. [47] demonstrated the general and efficient preparation of crystalline $2 \mathrm{D}$ boronate ester COF films (namely 2D BECOF-PP/BECOF-PN), which were composed of porphyrin macrocycles and phenyl/naphthyl linkers by employing the surfactant-monolayer-assisted interfacial synthesis on the water surface. The resultant 2D BECOF-PP was featured as free-standing thin films with large single-crystalline domains up to $60 \mathrm{~mm}^{2}$ and tunable thickness from 6 to $16 \mathrm{~nm}$.
Based on this single-crystalline COF film, they fabricated a neuromorphic memory device with a silicon nanowire-based lateral FET structure (Fig. 13a) and succeeded in emulating neuronal synaptic plasticity. The hysteresis behavior was observed for this neuromorphic device (Fig. 13b), which was compulsory for the memory effects including potentiation (learning), depression (erasing) and relaxation (forgetting) processes. In addition, the device held a fast response of $\sim 20 \mathrm{~s}$ for the potentiating saturation (Fig. 13c) and exhibited a depression time constant of $23 \mathrm{~s}$ in the forgetting period and $6 \mathrm{~s}$ in the erasing period (Fig. 13d). Moreover, the device showed a shortterm potentiation behavior, which presented a history-dependent memory and could mimic the intrinsic feature of neurons (Fig. 13e). This study opens the possibilities of integrating the COF-based CMs into neuromorphic computing, and also provides many opportunities for developing such emergent materials for flexible and wearable memory logic electronics.

More recently, Sun et al. [108] reported a surface-grown donor-acceptor type 2D polyimide COF film (namely PI-NT COF), which was synthesized from the condensation between the electron-donating $4,4^{\prime}, 4^{\prime \prime}$-triaminotriphenylamine and the electron-withdrawing naphthalene-1,4,5,8-tetracarboxylic dianhydride under solvothermal conditions. This 2D PI-NT COF film exhibited high crystallinity, good orientation preference, tunable thickness, and low surface roughness. The well-aligned donor and acceptor arrays in COF were beneficial for establishing the effective intramolecular charge transfer channels, which made the 2D PI-NT COF film a promising candidate for electronic applications. As a proof-of-concept, the memory devices based on 2D PINT COF films exhibited a typical nonvolatile resistive switching behavior at a threshold voltage of $+2.30 \mathrm{~V}$ on the positive scan and $-2.64 \mathrm{~V}$ on the negative scan. A high ON/OFF current ratio $\left(>10^{6}\right.$ for the positive scan and $10^{4}-$
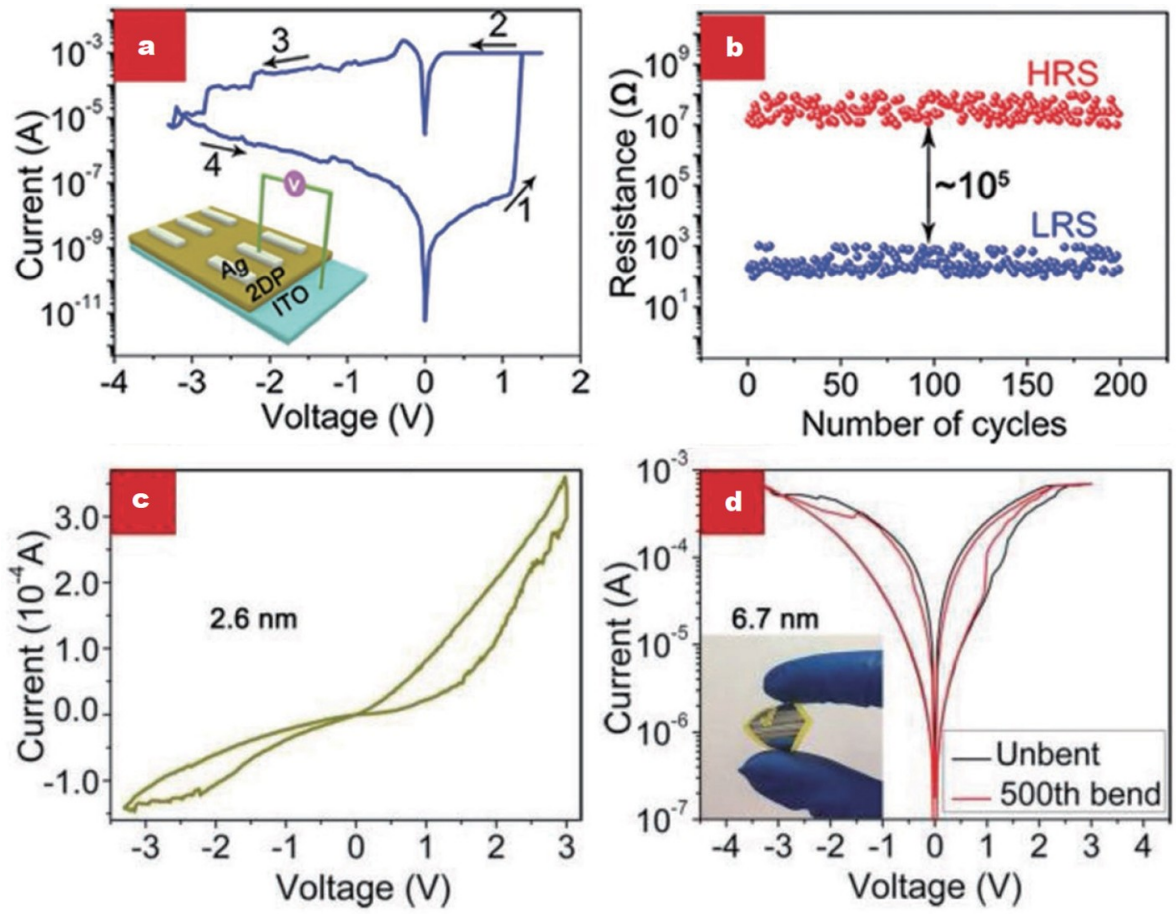

Figure 12 (a) $I-V$ characteristics for the initial formation process of the Ag/2DP/ITO memristor. (b) Endurance performance of the Ag/2DP/ITO memristor as a function of the cycling numbers. (c) $I-V$ characteristics of the Ag/2DP/graphene flexible memristor. (d) Switching curves of the Ag/2DP/graphene flexible memristor before and after repeated bending over 500 cycles. Reprinted with permission from Ref. [109], Copyright 2019, Wiley-VCH. 

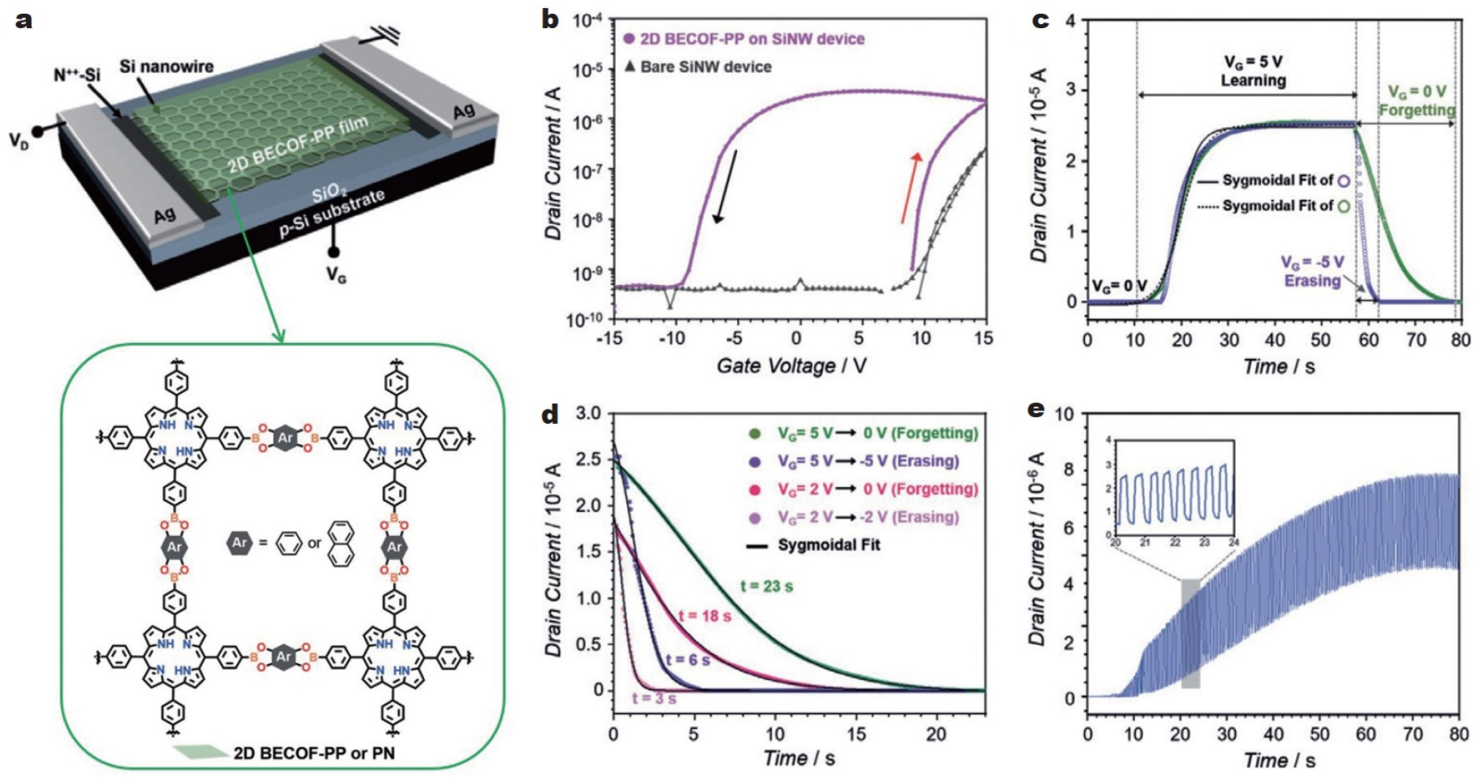

Figure 13 (a) Schematic illustration of the 2D BECOF-PP-based neuromorphic memory device with a silicon nanowire-based FET structure. (b) Transfer characteristics of the 2D BECOF-PP-based device. (c) Learn-erase/learn-forget cycles of the device. (d) History of the input signal in relaxation process of the device. (e) Short-term potentiation along with learning pulse of the device. Reprinted with permission from Ref. [47], Copyright 2020, Wiley-VCH.

$10^{6}$ for the negative scan) and long retention time $\left(>10^{4} \mathrm{~s}\right)$ were recorded. It could be assumed that when the flexible substrate was adopted, the flexible device would also maintain the reliable performance. This work provides an example that realizes the resistive memory properties of $2 \mathrm{D}$ crystalline $\mathrm{COF}$ materials based on their intrinsic charge transfer characteristics, which further demonstrates the promising application of COFs as information storage media.

\section{PEROVSKITES-BASED FLEXIBLE MEMRISTORS}

The intensive development of HPs-based CMs as active materials for optoelectronic devices such as solar cells, FETs, and light-emitting diodes [110-115] have been witnessed in recent years. It is also fascinating that there has been considerable research progress to pave the way for the application of HPs to information storage and neuromorphic computing [38,42,116123]. The $I-V$ hysteresis caused by the ion migration and the inconsistencies in the forward and reverse electric sweeps endow HPs with great potential for implementing memristors and artificial synaptic devices. Due to their exotic optical absorption, outstanding charge-transporting properties, and ultra-flexibility, HPs are believed as emerging contenders for next-generation electronic devices. Here, we focus on recent advances about HPbased flexible memristors [25,48,118,123-126].

In 2016, $\mathrm{Gu}$ et al. [48] prepared the organic-inorganic HP (OHP) of $\mathrm{CH}_{3} \mathrm{NH}_{3} \mathrm{PbI}_{3}$ film and adopted it as the active layer of flexible memristor (Fig. 14a). They found that a uniform perovskite layer could be formed on ITO-coated PET substrate, which exhibited reliable resistive switching characteristics with repeatable program/erase operations, long data retention over $10^{4} \mathrm{~s}$, and superior mechanical flexibility over 100 bending times (Fig. 14b). They suggested that the resistive switching was ascribed to the migration of vacancy defects and the formation of conducting filaments in the perovskite film (Fig. 14c). In 2017, Choi et al. [126] also fabricated a flexible memristor based on the OHP of $\mathrm{CH}_{3} \mathrm{NH}_{3} \mathrm{PbI}_{3}$. Intriguingly, they added hydro- iodic acid solution into the precursor solution of the OHP and found that the morphology of OHP film could be significantly enhanced for small grain size, low peak-to-valley depth and precise thickness, which served as an excellent insulating layer in the $\mathrm{Ag} / \mathrm{CH}_{3} \mathrm{NH}_{3} \mathrm{PbI}_{3} / \mathrm{Pt}$ device on a cyclo-olefin polymer (COP) substrate. The fabricated memristor afforded an electroformingfree resistive switching behavior with a high ON/OFF ratio of $10^{6}$ (Fig. 14d) and over 400 writing-erasing cycles (Fig. 14e) under an extremely low bending radius of $5.0 \mathrm{~mm}$, holding great promise for application in flexible and wearable electronic devices. Moreover, the migration of Ag ions from the top electrode was confirmed to account for the resistive switching (Fig. 14f). It is worth mentioning that Yan et al. [127] reported the first example of a free-standing fiber-shaped nonvolatile memristor device from $\mathrm{OHP}$ of $\mathrm{CH}_{3} \mathrm{NH}_{3} \mathrm{PbCl}_{x} \mathrm{I}_{3-x}$. The $\mathrm{CH}_{3} \mathrm{NH}_{3} \mathrm{PbCl}_{x} \mathrm{I}_{3-x}$ layer was deposited on the cylinder surface of a $\mathrm{TiO}_{2}$-coated titanium wire (with a diameter of $250 \mu \mathrm{m}$ ), and then the electrochemically inert $\mathrm{Au}$ wire (with a diameter of $25 \mu \mathrm{m}$ ) was chosen as the top electrode, leading to a memristor structured with $\mathrm{Au} / \mathrm{CH}_{3} \mathrm{NH}_{3} \mathrm{PbCl}_{x} \mathrm{I}_{3-x} / \mathrm{TiO}_{2} / \mathrm{Ti}$. The as-fabricated fiber-shaped perovskite memristor exhibited a bipolar resistive switching feature with small switching voltages of $+1.00 /-1.58 \mathrm{~V}$ and good retention stability over $2.0 \times 10^{4} \mathrm{~s}$, indicating the potential of OHPs for wearable memory devices and e-textiles.

In addition to the OHPs, all-inorganic HPs (IHPs) have also emerged as functional materials for memristor applications [128-131]. Han et al. [131] synthesized air-stable all-inorganic $\mathrm{CsPbI}_{3}$ perovskites and fabricated an Ag/polymethylmethacrylate (PMMA)/CsPbI $/ \mathrm{Pt}$ device on a flexible COP substrate. This flexible memristor afforded reproducible bipolar switching characteristics with an ultralow operating voltage below $0.2 \mathrm{~V}$, high ON/OFF ratio over $1.0 \times 10^{6}$, and multilevel data storage, along with outstanding mechanical flexibility. They ascribed the resistive switching to the conducting filaments formed by Ag cation migration through the lattice of the perovskite. Intrudingly, Yang et al. [124] demon- 

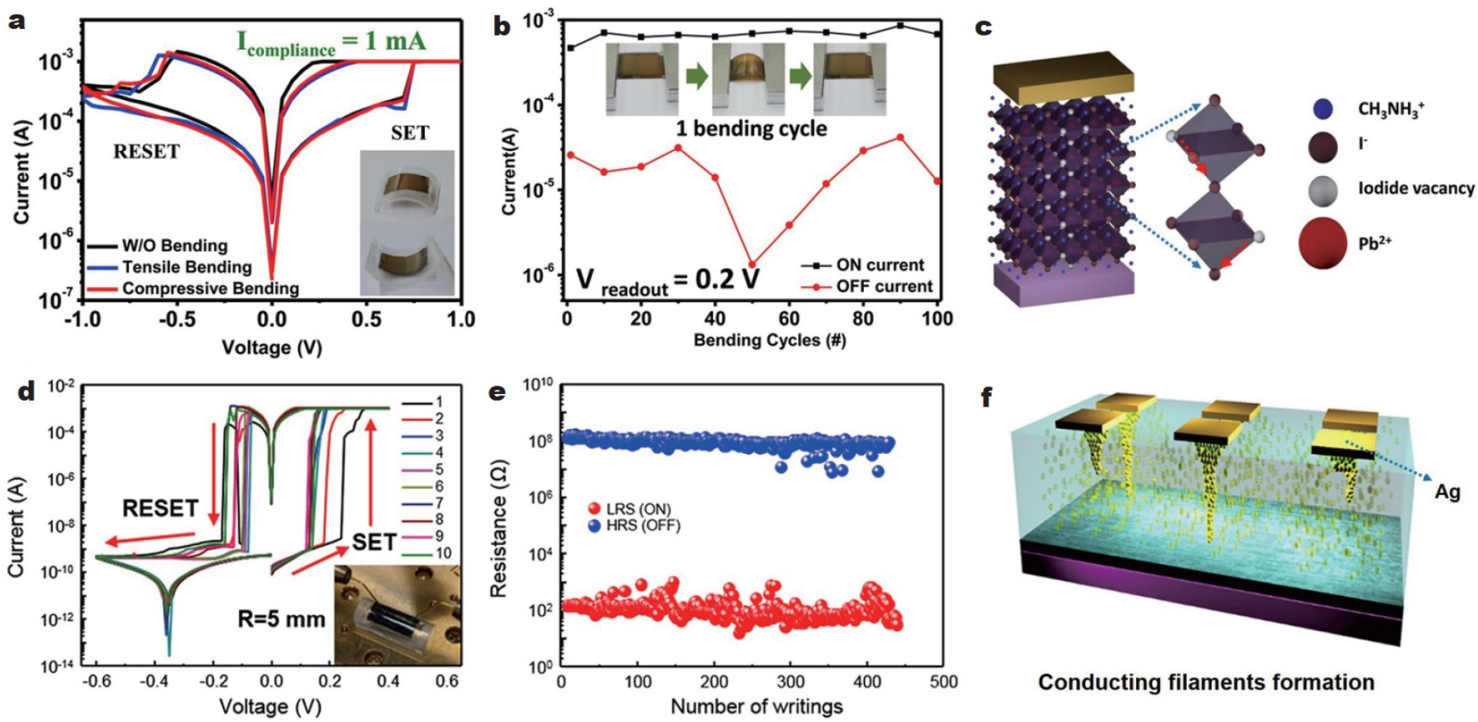

Figure 14 (a) $I-V$ characteristics of the flexible $\mathrm{Au} / \mathrm{CH}_{3} \mathrm{NH}_{3} \mathrm{PbI}_{3} / \mathrm{ITO}$ memristor without and with bending stress (a bending radius of $1.5 \mathrm{~cm}$ ). (b) Bending stability of the flexible Au/ $\mathrm{CH}_{3} \mathrm{NH}_{3} \mathrm{PbI}_{3} / \mathrm{ITO}$ memristor with repetitive bending cycles. (c) Proposed resistive switching mechanism of the flexible Au/ $\mathrm{CH}_{3} \mathrm{NH}_{3} \mathrm{PbI}_{3} / \mathrm{ITO}$ memristor. Reprinted with permission from Ref. [48], Copyright 2016, American Chemical Society. (d) I-V characteristics of the flexible $\mathrm{Ag} / \mathrm{CH}_{3} \mathrm{NH}_{3} \mathrm{PbI}_{3} / \mathrm{Pt}$ memristor under the bending radius of $5.0 \mathrm{~mm}$. (e) Endurance properties the flexible $\mathrm{Ag} / \mathrm{CH}_{3} \mathrm{NH}_{3} \mathrm{PbI} / \mathrm{Pt}$ memristor under the bending radius of $5.0 \mathrm{~mm}$. (f) Proposed resistive switching mechanism of the flexible $\mathrm{Ag} / \mathrm{CH}_{3} \mathrm{NH}_{3} \mathrm{PbI}_{3} / \mathrm{Pt}$ memristor. Reprinted with permission from Ref. [126], Copyright 2017, American Chemical Society.

strated a first example of highly transparent and flexible inorganic $\mathrm{CsPbCl}_{3}$-based photonic artificial synaptic device (Fig. 15a). They exposed the photonic artificial device with the "U" pattern (7 out of 9 pixels) under different ultraviolet (UV) light intensities from 1.25 to $25 \mu \mathrm{W} \mathrm{cm}{ }^{-2}$. The measured postsynaptic currents (PSCs) under different light intensities and light illumination time periods are shown in Fig. 15b. It can be seen that the PSC gradually enlarged with the increased light intensities from 1.25 to $25 \mu \mathrm{W} \mathrm{cm}$ cm $^{-2}$ and prolonged illumination time from 0.3 to $5 \mathrm{~s}$, which indicated an enhanced memory behavior. In biological neuron networks, the stronger link between neurons can be generated by the larger stimulation. In their study, the regulation of this photonic artificial synapse clearly revealed the photo-recording functionality, which well mimicked the biological neuron systems. This optically triggered artificial synapse showed distinguished synaptic characteristics such as paired-pulse facilitation, short/long-term memory, and synaptic behavior analogous to the iris in the human eye, making it possible to implement optical data storage, neuromorphic computing, and bio-inspired smart visual recognition.

It should be noted that the above perovskites contain toxic $\mathrm{Pb}$ element, which may restrict their further practical applications. To this regard, numerous research efforts have been devoted to developing lead-free perovskites for memristor devices [25,58,122]. As shown in Fig. 16a, Hu et al. [122] successfully synthesized nontoxic and stable ultrathin $\mathrm{Cs}_{3} \mathrm{Bi}_{2} \mathrm{I}_{9}$ nanosheets and explored their potential applications in flexible resistive memory devices. The as-prepared flexible memristors based on such ultrathin $\mathrm{Cs}_{3} \mathrm{Bi}_{2} \mathrm{I}_{9}$ nanosheets presented excellent environmental stability under flat and bent conditions (Fig. 16b). Moreover, they displayed typical bipolar resistive switching behavior and remarkable mechanical flexibility over 100 bending cycles (Fig. 16c). As another typical example, Ye et al. [58] reported a lead-free $\mathrm{AgBiI}_{4}$ perovskite-based memristor with exalted electrical performance (Fig. 16d). They fabricated a flexible $\mathrm{AgBiI}_{4}$ device on polyethylene naphthalate (PEN) substrate and examined its mechanical reliability in ambient air condition. The relevant flexible memristor displayed outstanding mechanical stability over 1000 cycles under repeated bending tests (Fig. 16e). In addition, this memristor afforded excellent characteristics such as an ultralow operating voltage of $\sim 0.16 \mathrm{~V}$ and a high ON/OFF ratio of $\sim 10^{4}$ compared with other reported perovskite-based memory devices (Fig. 16f). According to the $I-V$ responses and the TEM measurements, they proposed the resistive switching was caused by the formation and disruption of the $\mathrm{Ag}$ filaments. Given these aforementioned research endeavors, we believe that the exceptional characteristics of perovskites-based flexible memristors can actuate the potential for realizing next-generation information storage and intelligent processing systems.

\section{CONCLUSIONS AND PROSPECTIVE}

In conclusion, this review provides a timely summary of recent progress in CMs for flexible memristor applications. The two representative memristive device structures and switching mechanisms are briefly introduced. The main content focuses on the 2D materials, MOFs, COFs, and perovskites, which have been demonstrated as promising CMs for the development of flexible and wearable memristors (Table 1). Moreover, their potential for high-performance data storage and artificial synapses is presented. Notably, the data storage and neuromorphic devices are two important application fields for CMsbased flexible memristors. The summary and prospective related to these two fields are discussed as below.

\section{Data storage application}

With the advent of artificial intelligence and Internet of Things, high-performance data storage technologies with lower power consumption, larger storage capacity, faster switching rate, and higher integration density have become an inevitable trend 


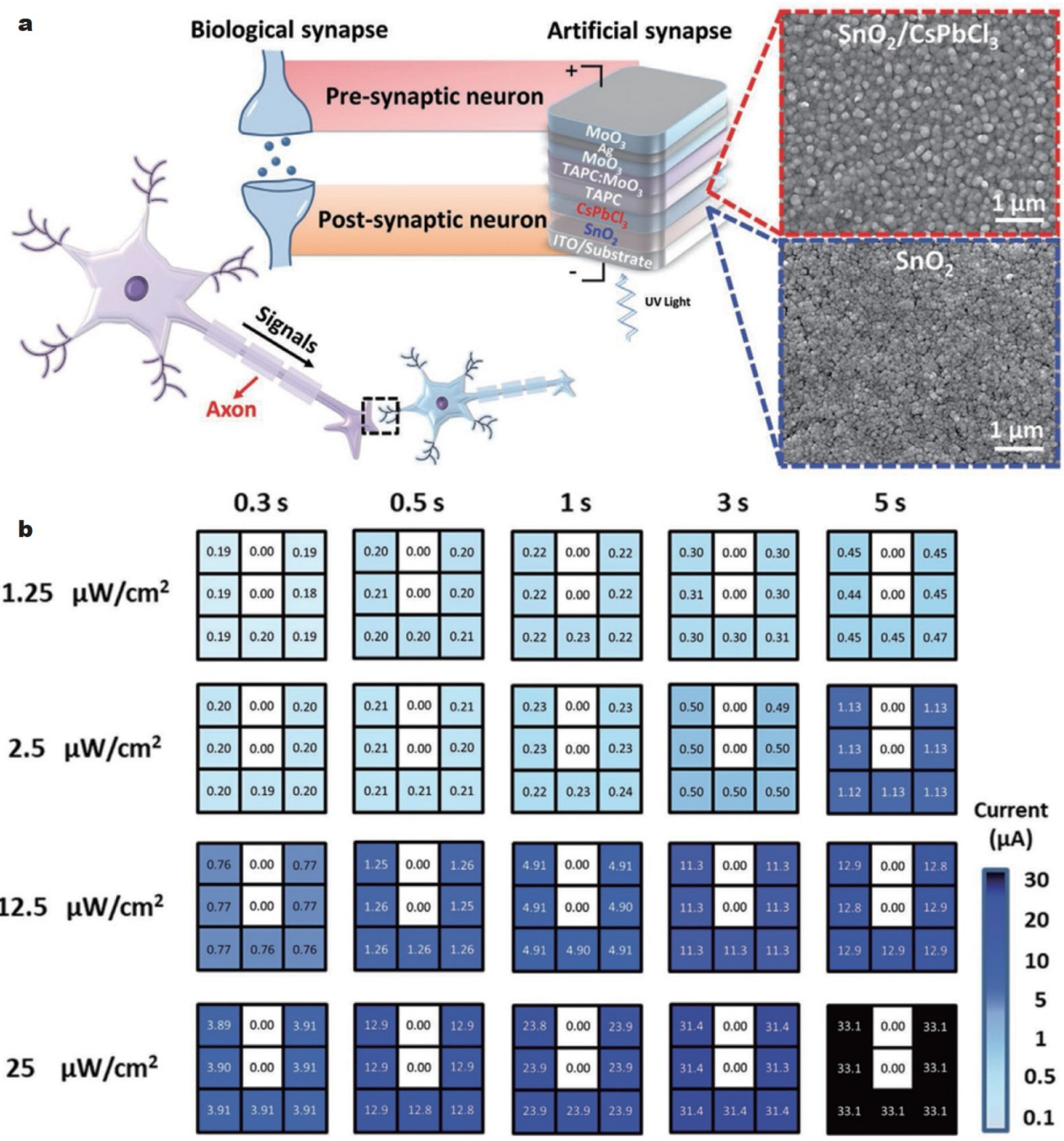

Figure 15 (a) Schematic illustration of biological synapse in the human brain and the artificial synapse, including the biological synapses and neurons, the artificial two-terminal $\mathrm{CsPbCl}_{3}$-based device and SEM images of $\mathrm{SnO}_{2}$ and $\mathrm{SnO}_{2} / \mathrm{CsPbCl}_{3}$. (b) The input images in the "U" pattern that consists of $3 \times 3$ pixels encoded by various light intensities from 1.25 to $25 \mu \mathrm{W} \mathrm{cm}$-2 and light illumination time periods from 0.3 to $5 \mathrm{~s}$. The number in each pixel represents the measured PSC value. Reprinted with permission from Ref. [124], Copyright 2020, Wiley-VCH.

[30,67]. Moreover, the flexible and wearable electronics have aroused particular attention due to their potential for implementing intelligent human-computer interaction [67]. Therefore, the low-power and high-density flexible memristors are regarded as an ideal platform to realize the goals of next-generation information technology. As memory electronics, the CMs-based flexible memristors can afford low power consumption $(<100 \mathrm{fJ})$, fast switching response $(<100 \mathrm{~ns})$, highdensity data storage, 3D memory stacking architecture, and simple device preparation techniques, which promise a bright future for long-term and high-efficiency mass data storage application.

\section{Neuromorphic device application}

The human brain, which contains about $10^{11}$ neurons and $10^{15}$ synapses, is considered as the most intelligent computing system [132]. Inspired by the structure and functions of biological synapses and neurons, memristor-based neuromorphic devices have been proposed for constructing artificial neural networks [20], which are aimed at achieving neuromorphic computing like the human brain. As flexible synaptic devices, the CMsbased analog memristors not only succeed in mimicing the data processing and memory functions of biological synapses, but also hold promise to implement intelligent perception (e.g., biorobots) and artificial sensory systems (e.g., human-machine integration). Thus, the development of CMs-based flexible neuromorphic devices opens up many possibilities for the realization of next-generation computing platforms with high comfort and conformability to the biological systems.

On the basis of the recent encouraging research progress in data storage and neuromorphic devices, it can be anticipated that the CMs-based memristors will provide fascinating opportunities toward the artificial intelligent era. Nevertheless, the development of CMs-based flexible memristors still remains at the early stage. Some challenges and issues need to be tackled to further push their practical applications. From the standpoint of material level, since the switching characteristics and reliability of the flexible memristors are strongly correlated with the active materials, their ease of preparation, diversity, and stability deserve more attention for satisfying the robust output perfor- 

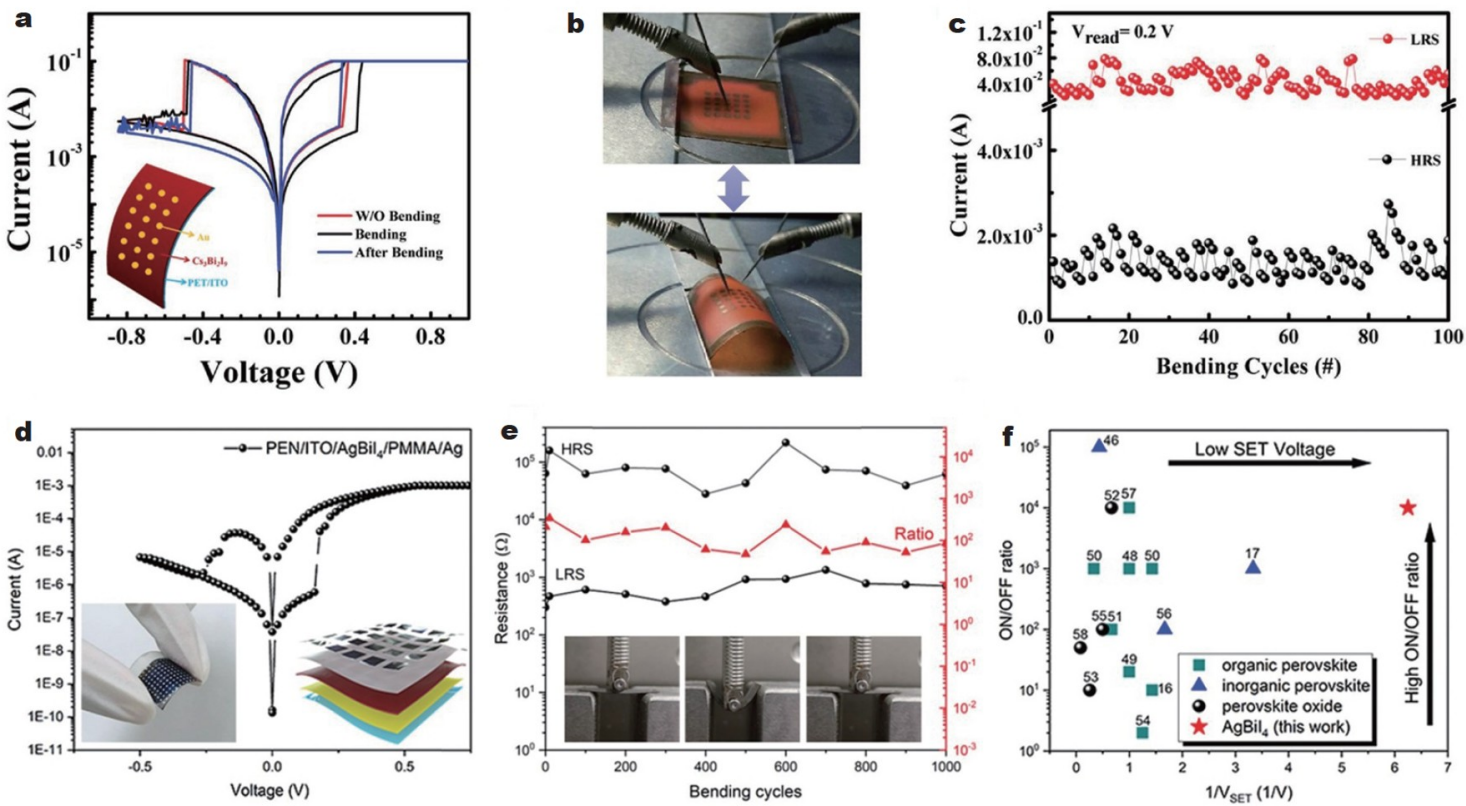

Figure 16 (a) $I-V$ characteristics of the flexible $\mathrm{Au} / \mathrm{Cs}_{3} \mathrm{Bi}_{2} \mathrm{I}_{9} / \mathrm{ITO} / \mathrm{PET}$ memristor without and with bending stress (a bending radius of $0.9 \mathrm{~cm}$ ). (b) Photographs of the flexible $\mathrm{Au} / \mathrm{Cs}_{3} \mathrm{Bi}_{2} \mathrm{I}_{9} / \mathrm{ITO} / \mathrm{PET}$ memristor during one bending cycle procedure. (c) Endurance test of the flexible Au/Cs $\mathrm{Bi}_{2} \mathrm{I}_{9} / \mathrm{ITO} / \mathrm{PET}$ memristor with repeating 100 bending cycles. Reprinted with permission from Ref. [122], Copyright 2017, Wiley-VCH. (d) $I-V$ characteristics of the flexible Ag/PMMA/AgBiI $/$ /TTO memristor. (e) Repeated bending stability of the flexible Ag/PMMA/AgBiI $/$ /TO memristor. (f) The ON/OFF ratio of the flexible Ag/ PMMA/AgBiI $/$ /ITO memristor compared with other reported perovskites-based devices (the numbers refer to the cited references in Ref. [58]). Reprinted with permission from Ref. [58], Copyright 2020, The Royal Society of Chemistry.

Table 1 Summary of the flexible memristor performance based on various CMs

\begin{tabular}{|c|c|c|c|c|c|c|}
\hline Device structure & Category & Flexible substrate & Current ratio & Bending cycles & Other features & Ref. \\
\hline $\mathrm{Al} / \mathrm{GO} / \mathrm{Al}$ & $2 \mathrm{D}$ materials & Polyethers-ulfone & $10^{2}$ & $10^{3}$ & Retention time of $10^{5} \mathrm{~s}$ & [45] \\
\hline $\mathrm{Al} / \mathrm{GO}-\mathrm{TiO}_{2} / \mathrm{ITO}$ & 2D materials & PET & $>10$ & $10^{4}$ & Bending radius of $1.27 \mathrm{~cm}$ & [74] \\
\hline Graphene/ $\mathrm{MoS}_{2-x} \mathrm{O}_{x} /$ Graphene & 2D materials & Polyimide & $>10$ & $1.2 \times 10^{3}$ & Bending radius of $1 \mathrm{~cm}$ & [8] \\
\hline Ag/BPQD-PVP/Au & 2D materials & PET & $6 \times 10^{4}$ & - & Retention time of $1.1 \times 10^{3} \mathrm{~s}$ & {$[72]$} \\
\hline $\mathrm{Au} / \mathrm{h}-\mathrm{BN} /$ graphene/h-BN/Ag & $2 \mathrm{D}$ materials & PET & $10^{3}$ & - & Writing voltage window $>4 \mathrm{~V}$ & {$[51]$} \\
\hline $\mathrm{Al} / \mathrm{Ti}_{3} \mathrm{C}_{2} \mathrm{~T}_{x} / \mathrm{ITO}$ & $2 \mathrm{D}$ materials & PET & $1: 10^{2.7}: 10^{4.1}$ & $5 \times 10^{3}$ & Bending radius of $2.1 \mathrm{~cm}$ & [86] \\
\hline $\mathrm{Au} / \mathrm{HKUST}-1 / \mathrm{Au}$ & MOFs & PET & $>10$ & $3 \times 10^{2}$ & Stretching strain of $2 \%$ & [98] \\
\hline Ag/MIL-53/GaInSn@PDMS & MOFs & GaInSn@PDMS & $2 \times 10^{2}$ & 50 & Stretching strain of $10 \%$ & [93] \\
\hline $\mathrm{Ag} / \mathrm{ZIF}-8 / \mathrm{Au}$ & MOFs & PET & $10^{6}$ & - & Bending radius of $1.42 \mathrm{~cm}$ & [46] \\
\hline $\mathrm{Al} / \mathrm{ZIF}-8 / \mathrm{Au}$ & MOFs & PET & $10^{3}$ & $>10^{2}$ & Bending radius of $1.35 \mathrm{~cm}$ & [96] \\
\hline rGO/MoS ${ }_{2} @ Z I F-8 / r G O$ & 2D materials@MOFs & PET & $7 \times 10^{4}$ & - & Retention time of $1.5 \times 10^{3} \mathrm{~s}$ & {$[100]$} \\
\hline $\mathrm{Ag} / 2 \mathrm{DP} /$ Graphene & COFs & Polyimide & $>10$ & $5 \times 10^{2}$ & 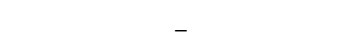 & [109] \\
\hline $\mathrm{Au} / \mathrm{CH}_{3} \mathrm{NH}_{3} \mathrm{PbI}_{3} / \mathrm{ITO}$ & OHPs & PET & $>10$ & $10^{2}$ & Bending radius of $1.5 \mathrm{~cm}$ & {$[48]$} \\
\hline $\mathrm{Ag} / \mathrm{CH}_{3} \mathrm{NH}_{3} \mathrm{PbI}_{3} / \mathrm{Pt}$ & OHPs & $\mathrm{COP}$ & $10^{6}$ & - & Bending radius of $0.5 \mathrm{~cm}$ & {$[126]$} \\
\hline $\mathrm{Au} / \mathrm{CH}_{3} \mathrm{NH}_{3} \mathrm{PbCl}_{x} \mathrm{I}_{3-x} / \mathrm{TiO}_{2} / \mathrm{Ti}$ & OHPs & Ti wire & 20 & - & Retention time of $2.5 \times 10^{4} \mathrm{~s}$ & {$[127]$} \\
\hline $\mathrm{Ag} / \mathrm{PMMA} / \mathrm{CsPbI}_{3} / \mathrm{Pt}$ & IHPs & $\mathrm{COP}$ & $10^{6}$ & - & Bending radius of $0.5 \mathrm{~cm}$ & {$[131]$} \\
\hline $\mathrm{Au} / \mathrm{Cs}_{3} \mathrm{Bi}_{2} \mathrm{I}_{9} / \mathrm{ITO}$ & IHPs & PET & $10^{2}$ & $>10^{2}$ & Bending radius of $0.9 \mathrm{~cm}$ & {$[122]$} \\
\hline $\mathrm{Ag} / \mathrm{PMMA} / \mathrm{AgBiI}_{4} / \mathrm{ITO}$ & IHPs & PEN & $>10^{2}$ & $>10^{3}$ & - & {$[58]$} \\
\hline
\end{tabular}

mances: (1) for 2D materials, the synthetic methods of various functionalized 2D materials such as the solution-processing or the chemical vapor deposition techniques on diverse folding and stretching substrates require more efforts to guarantee the uniformity before device fabrication. The functional groups attached onto $2 \mathrm{D}$ materials (e.g., GO) can greatly modify their electronic properties, and deserve more systematic investigations. Moreover, the physical and chemical properties of different $2 \mathrm{D}$ materials are distinguished from each other. Thus, one research direction is to integrate different $2 \mathrm{D}$ materials into a single system (e.g., layered stacking), and fully explore their potential for high-performance flexible memristors. (2) For 
MOFs- and COFs-based materials, the current synthetic methods are mainly restrained in the laboratory due to the relatively harsh conditions. Thus, simpler and more efficient synthetic strategies are still waiting to be proposed for the large-scale production. In addition, to improve the stability of these porous materials, it is necessary to exploit novel building blocks with enhanced structural robustness and controllability. Moreover, although some MOFs and COFs have been demonstrated as potential candidates for resistive memory, many of these materials still exhibited insufficient electrical properties, which hinder the further application in electronic devices. Bearing this in mind, the effective ways for improving their conductive ability are important to be developed. (3) For perovskites, the environmental and thermodynamic stability are two main issues that restrict their development and application. The factors of moisture, oxygen, and heat can result in the degradation of materials, which suppress the air stability of perovskite-based memristors. Therefore, the perovskites with long-term environmental reliability should be focused on for further practical application. Overall, since the aforementioned diverse materials usually hold different physical and electronic properties, it is also very important to figure out the underlying switching mechanisms in different cases with the help of theoretical and experimental demonstrations.

From the standpoint of device level, although the prototype memory logic and synaptic devices with long retention and analog responses have been reported, the existing issues of switching non-reproducibility, cycle-to-cycle/device-to-device variations under continuous bending operation and the incompatibility with the CMOS technology need to be particularly addressed. In general, the device performance is closely associated with the active materials and the device structure. The crystallinity, ordering, stability, and mechanical flexibility of CMs play a key role in determining the switching reliability and batch-to-batch reproducibility under consecutive bending operations. Meanwhile, adopting the device structure that conforms to the conventional CMOS technology can further accelerate the application of flexible memristors in the future. The flexible design and preparation of CMs is a crucial requirement for wearable and smart electronics. However, several CMs confront the challenge of holding enough mechanical flexibility for flexible applications. To this regard, more efficient strategies should be developed for designing materials and structures to achieve excellent mechanical flexibility. The potential approaches such as preparing intrinsically soft and stretchable materials, and properly buckling the fabricated flexible devices could be considered. Additionally, the structureproperty relations, i.e., how the material structure, the solid-state packing, and the film preparation technique influence the mechanical bending and ultimate flexible device performance need vast investigations, in order to provide guidelines for rational material design. In this aspect, the development of highefficiency and high-elasticity CMs should be brought to the forefront. Moreover, toward the implementation of the novel logic/neuromorphic hardware and real-time monitoring wearable electronics, the leaps from laboratory to industrialization deserve more attention and endeavors. It is worth bringing academic and industrial communities closer than ever to launch a new round of information technology revolution.

Envisaging the future, attributed to the merits of good ordering and few defects, CMs-based memristors pave the way for achieving reliable device integration and function exploitation, which sheds light on the promise of in-memory computing and artificial neural networks. Considering that the traditional von Neumann computer systems are confronted with the bottleneck of competing complicated tasks of learning, judging and decision making, the neuromorphic devices and technologies based on memristors seem to be more exciting. Therefore, although there exist numerous difficulties to surmount, there are sound reasons to believe that the development and integration of CMs-based flexible memristors is a promising field for nextgeneration information computing and artificial intelligent applications.

Received 29 June 2021; accepted 12 August 2021; published online 28 September 2021

1 Chua L. Memristor-The missing circuit element. IEEE Trans Circuit Theor, 1971, 18: 507-519

2 Strukov DB, Snider GS, Stewart DR, et al. The missing memristor found. Nature, 2008, 453: 80-83

3 Hus SM, Ge R, Chen PA, et al. Observation of single-defect memristor in an $\mathrm{MoS}_{2}$ atomic sheet. Nat Nanotechnol, 2021, 16: 58-62

4 Sun B, Zhou G, Guo T, et al. Biomemristors as the next generation bioelectronics. Nano Energy, 2020, 75: 104938

5 Wang TY, Meng JL, Rao MY, et al. Three-dimensional nanoscale flexible memristor networks with ultralow power for information transmission and processing application. Nano Lett, 2020, 20: 41114120

6 Wang TY, Meng JL, Chen L, et al. Flexible 3D memristor array for binary storage and multi-states neuromorphic computing applications. InfoMat, 2021, 3: 212-221

7 Chen F, Zhou Y, Zhu Y, et al. Recent progress in artificial synaptic devices: Materials, processing and applications. J Mater Chem C, 2021 , 9: 8372-8394

8 Wang M, Cai S, Pan C, et al. Robust memristors based on layered twodimensional materials. Nat Electron, 2018, 1: 130-136

9 Goswami S, Matula AJ, Rath SP, et al. Robust resistive memory devices using solution-processable metal-coordinated azo aromatics. Nat Mater, 2017, 16: 1216-1224

10 Zhang Z, Wang Z, Shi T, et al. Memory materials and devices: From concept to application. InfoMat, 2020, 2: 261-290

11 Chen Y, Liu G, Wang C, et al. Polymer memristor for information storage and neuromorphic applications. Mater Horiz, 2014, 1: 489

12 Zhang B, Chen W, Zeng J, et al. 90\% yield production of polymer nano-memristor for in-memory computing. Nat Commun, 2021, 12 1984

13 Sun K, Chen J, Yan X. The future of memristors: Materials engineering and neural networks. Adv Funct Mater, 2020, 31: 2006773

14 Zhong Y, Tang J, Li X, et al. Dynamic memristor-based reservoir computing for high-efficiency temporal signal processing. Nat Commun, 2021, 12: 408

15 Yao P, Wu H, Gao B, et al. Fully hardware-implemented memristor convolutional neural network. Nature, 2020, 577: 641-646

16 Huang HM, Wang Z, Wang T, et al. Artificial neural networks based on memristive devices: From device to system. Adv Intelligent Syst, 2020, 2: 2000149

17 Wang Z, Joshi S, Savel'ev SE, et al. Memristors with diffusive dynamics as synaptic emulators for neuromorphic computing. Nat Mater, 2017, 16: $101-108$

18 Prezioso M, Merrikh-Bayat F, Hoskins BD, et al. Training and operation of an integrated neuromorphic network based on metal-oxide memristors. Nature, 2015, 521: 61-64

19 Kang DH, Kim JH, Oh S, et al. A neuromorphic device implemented on a salmon-DNA electrolyte and its application to artificial neural networks. Adv Sci, 2019, 6: 1901265

20 Choi S, Yang J, Wang G. Emerging memristive artificial synapses and neurons for energy-efficient neuromorphic computing. Adv Mater, 
2020, 32: 2004659

21 Sun F, Lu Q, Feng S, et al. Flexible artificial sensory systems based on neuromorphic devices. ACS Nano, 2021, 15: 3875-3899

22 Huang L, Diao J, Nie H, et al. Memristor based binary convolutional neural network architecture with configurable neurons. Front Neurosci, 2021, 15: 639526

23 Zhu K, Liang X, Yuan B, et al. Graphene-boron nitride-graphene cross-point memristors with three stable resistive states. ACS Appl Mater Interfaces, 2019, 11: 37999-38005

24 Li Y, Zhang C, Ling S, et al. Toward highly robust nonvolatile multilevel memory by fine tuning of the nanostructural crystalline solidstate order. Small, 2021, 17: 2100102

25 Fang Y, Zhai S, Chu L, et al. Advances in halide perovskite memristor from lead-based to lead-free materials. ACS Appl Mater Interfaces, 2021, 13: 17141-17157

26 Zhang L, Gong $\mathrm{T}$, Wang $\mathrm{H}$, et al. Memristive devices based on emerging two-dimensional materials beyond graphene. Nanoscale, 2019, 11: 12413-12435

$27 \mathrm{Yu} \mathrm{T}, \mathrm{He} \mathrm{F}$, Zhao J, et al. $\mathrm{Hf}_{0.5} \mathrm{Zr}_{0.5} \mathrm{O}_{2}$-based ferroelectric memristor with multilevel storage potential and artificial synaptic plasticity. Sci China Mater, 2020, 64: 727-738

28 Yao Z, Pan L, Liu L, et al. Simultaneous implementation of resistive switching and rectifying effects in a metal-organic framework with switched hydrogen bond pathway. Sci Adv, 2019, 5: eaaw4515

29 Zhou G, Sun B, Hu X, et al. Negative photoconductance effect: An extension function of the $\mathrm{TiO}_{x}$-based memristor. Adv Sci, 2021, 8: 2003765

30 Li Y, Qian Q, Zhu X, et al. Recent advances in organic-based materials for resistive memory applications. InfoMat, 2020, 2: 995-1033

31 Zhou G, Ren Z, Wang L, et al. Artificial and wearable albumen protein memristor arrays with integrated memory logic gate functionality. Mater Horiz, 2019, 6: 1877-1882

32 Zhang B, Fan F, Xue W, et al. Redox gated polymer memristive processing memory unit. Nat Commun, 2019, 10: 736

33 Zhao YY, Sun WJ, Wang J, et al. All-inorganic ionic polymer-based memristor for high-performance and flexible artificial synapse. Adv Funct Mater, 2020, 30: 2004245

34 Lan J, Cao G, Wang J, et al. Artificial nociceptor based on $\mathrm{TiO}_{2}$ nanosheet memristor. Sci China Mater, 2021, 64: 1703-1712

35 Sivan M, Li Y, Veluri $\mathrm{H}$, et al. All WSe $\mathrm{H}_{2}$ 1T1R resistive ram cell for future monolithic 3D embedded memory integration. Nat Commun, 2019, 10: 5201

36 Chen T, Yang S, Wang J, et al. Flexible artificial memristive synapse constructed from solution-processed $\mathrm{MgO}$-graphene oxide quantum dot hybrid films. Adv Electron Mater, 2021, 7: 2000882

37 Zhou Z, Xiu F, Jiang T, et al. Solution-processable zinc oxide nanorods and a reduced graphene oxide hybrid nanostructure for highly flexible and stable memristor. J Mater Chem C, 2019, 7: 10764-10768

38 Zhao $\mathrm{X}, \mathrm{Xu} \mathrm{H}$, Wang $\mathrm{Z}$, et al. Memristors with organic-inorganic halide perovskites. InfoMat, 2019, 1: inf2.12012

39 Wang Y, Sun L, Wang C, et al. Organic crystalline materials in flexible electronics. Chem Soc Rev, 2019, 48: 1492-1530

40 Ma Y, Zhang Y, Cai S, et al. Flexible hybrid electronics for digital healthcare. Adv Mater, 2020, 32: 1902062

41 Zhou L, Mao J, Ren Y, et al. Recent advances of flexible data storage devices based on organic nanoscaled materials. Small, 2018, 14: 1703126

42 Kang K, Ahn H, Song Y, et al. High-performance solution-processed organo-metal halide perovskite unipolar resistive memory devices in a cross-bar array structure. Adv Mater, 2019, 31: 1804841

43 Son D, Chae SI, Kim M, et al. Colloidal synthesis of uniform-sized molybdenum disulfide nanosheets for wafer-scale flexible nonvolatile memory. Adv Mater, 2016, 28: 9326-9332

44 Qian Y, Zhang X, Xie L, et al. Stretchable organic semiconductor devices. Adv Mater, 2016, 28: 9243-9265

45 Jeong HY, Kim JY, Kim JW, et al. Graphene oxide thin films for flexible nonvolatile memory applications. Nano Lett, 2010, 10: 43814386

46 Liu Y, Wang H, Shi W, et al. Alcohol-mediated resistance-switching behavior in metal-organic framework-based electronic devices. Angew Chem Int Ed, 2016, 55: 8884-8888

47 Park SW, Liao Z, Ibarlucea B, et al. Two-dimensional boronate ester covalent organic framework thin films with large single crystalline domains for a neuromorphic memory device. Angew Chem Int Ed, 2020, 59: 8218-8224

$48 \mathrm{Gu}$ C, Lee JS. Flexible hybrid organic-inorganic perovskite memory. ACS Nano, 2016, 10: 5413-5418

49 Linn E, Rosezin R, Kügeler C, et al. Complementary resistive switches for passive nanocrossbar memories. Nat Mater, 2010, 9: 403-406

$50 \mathrm{Vu} \mathrm{QA}$, Kim $\mathrm{H}$, Nguyen VL, et al. A high-on/off-ratio floating-gate memristor array on a flexible substrate via CVD-grown large-area 2D layer stacking. Adv Mater, 2017, 29: 1703363

51 Sun L, Zhang Y, Han G, et al. Self-selective van der Waals heterostructures for large scale memory array. Nat Commun, 2019, 10: 3161

52 Zhang J, Yang T, Wang J, et al. Memristive device with highly continuous conduction modulation and its underlying physical mechanism for electronic synapse application. Sci China Mater, 2021, 64: 179188

53 Rani A, Kim DH. A mechanistic study on graphene-based nonvolatile ReRAM devices. J Mater Chem C, 2016, 4: 11007-11031

54 Gabel M, Gu Y. Understanding microscopic operating mechanisms of a van der Waals planar ferroelectric memristor. Adv Funct Mater, 2020, 31: 2009999

55 Chen $\mathrm{K}$, Tsutsui M, Zhuge F, et al. Nanochannel-based interfacial memristor: Electrokinetic analysis of the frequency characteristics. Adv Electron Mater, 2021, 7: 2000848

56 Sokolov AS, Ali M, Riaz R, et al. Silver-adapted diffusive memristor based on organic nitrogen-doped graphene oxide quantum dots $(\mathrm{N}$ GOQDs) for artificial biosynapse applications. Adv Funct Mater, 2019, 29: 1807504

57 Lee SM, Choi J, Jeon JB, et al. Conducting bridge resistive switching behaviors in cubic $\mathrm{MAPbI}_{3}$, orthorhombic $\mathrm{RbPbI}_{3}$, and their mixtures. Adv Electron Mater, 2019, 5: 1800586

58 Ye H, Sun B, Wang Z, et al. High performance flexible memristors based on a lead free $\mathrm{AgBiI}_{4}$ perovskite with an ultralow operating voltage. J Mater Chem C, 2020, 8: 14155-14163

59 Jo SH, Chang T, Ebong I, et al. Nanoscale memristor device as synapse in neuromorphic systems. Nano Lett, 2010, 10: 1297-1301

60 Di Martino G, Demetriadou A, Li W, et al. Real-time in situ optical tracking of oxygen vacancy migration in memristors. Nat Electron, 2020, 3: 687-693

$61 \mathrm{Xu} \mathrm{W}$, Cho H, Kim YH, et al. Organometal halide perovskite artificial synapses. Adv Mater, 2016, 28: 5916-5922

62 Solanki A, Guerrero A, Zhang Q, et al. Interfacial mechanism for efficient resistive switching in Ruddlesden-Popper perovskites for non-volatile memories. J Phys Chem Lett, 2019, 11: 463-470

63 Zhou F, Liu $\mathrm{Y}$, Shen $\mathrm{X}$, et al. Low-voltage, optoelectronic $\mathrm{CH}_{3} \mathrm{NH}_{3} \mathrm{PbI}_{3-x} \mathrm{Cl}_{x}$ memory with integrated sensing and logic operations. Adv Funct Mater, 2018, 28: 1800080

64 Wang H, Yu T, Zhao J, et al. Low-power memristors based on layered 2D SnSe/graphene materials. Sci China Mater, 2021, 64: 1989-1996

65 Xiong W, Zhu LQ, Ye C, et al. Flexible poly(vinyl alcohol)-graphene oxide hybrid nanocomposite based cognitive memristor with Pavlovian-conditioned reflex activities. Adv Electron Mater, 2020, 6: 1901402

66 Chen Y, Gao G, Zhao J, et al. Piezotronic graphene artificial sensory synapse. Adv Funct Mater, 2019, 29: 1900959

67 Bertolazzi S, Bondavalli P, Roche S, et al. Nonvolatile memories based on graphene and related 2D materials. Adv Mater, 2019, 31: 1806663

68 Tan C, Liu Z, Huang W, et al. Non-volatile resistive memory devices based on solution-processed ultrathin two-dimensional nanomaterials. Chem Soc Rev, 2015, 44: 2615-2628

69 Huh W, Lee D, Lee CH. Memristors based on 2D materials as an artificial synapse for neuromorphic electronics. Adv Mater, 2020, 32 2002092

70 Khot AC, Dongale TD, Park JH, et al. $\mathrm{Ti}_{3} \mathrm{C}_{2}$-based MXene oxide nanosheets for resistive memory and synaptic learning applications. ACS Appl Mater Interfaces, 2021, 13: 5216-5227 
71 Cao Y, Tian X, Gu J, et al. Covalent functionalization of black phosphorus with conjugated polymer for information storage. Angew Chem Int Ed, 2018, 57: 4543-4548

72 Zhang X, Xie H, Liu Z, et al. Black phosphorus quantum dots. Angew Chem Int Ed, 2015, 54: 3653-3657

73 Liu T, Wu W, Liao KN, et al. Fabrication of carboxymethyl cellulose and graphene oxide bio-nanocomposites for flexible nonvolatile resistive switching memory devices. Carbohydrate Polyms, 2019, 214: 213-220

74 Zhao X, Wang Z, Xie Y, et al. Photocatalytic reduction of graphene oxide- $\mathrm{TiO}_{2}$ nanocomposites for improving resistive-switching memory behaviors. Small, 2018, 14: 1801325

75 Liu J, Yin Z, Cao X, et al. Fabrication of flexible, all-reduced graphene oxide non-volatile memory devices. Adv Mater, 2013, 25: 233-238

76 Schranghamer TF, Oberoi A, Das S. Graphene memristive synapses for high precision neuromorphic computing. Nat Commun, 2020, 11: 5474

77 Tian H, Deng B, Chin ML, et al. A dynamically reconfigurable ambipolar black phosphorus memory device. ACS Nano, 2016, 10: 10428-10435

78 Xiang D, Liu T, Xu J, et al. Two-dimensional multibit optoelectronic memory with broadband spectrum distinction. Nat Commun, 2018, 9: 2966

79 Wu X, Ge R, Chen PA, et al. Thinnest nonvolatile memory based on monolayer h-BN. Adv Mater, 2019, 31: 1806790

80 Wang Y, Gong Y, Yang L, et al. MXene-ZnO memristor for multimodal in-sensor computing. Adv Funct Mater, 2021, 31: 2100144

81 Wang K, Chen J, Yan X. MXene $\mathrm{Ti}_{3} \mathrm{C}_{2}$ memristor for neuromorphic behavior and decimal arithmetic operation applications. Nano Energy, 2021, 79: 105453

82 Shahzad F, Iqbal A, Kim $\mathrm{H}$, et al. 2D transition metal carbides (MXenes): Applications as an electrically conducting material. Adv Mater, 2020, 32: 2002159

83 Han M, Shuck CE, Rakhmanov $\mathrm{R}$, et al. Beyond $\mathrm{Ti}_{3} \mathrm{C}_{2} \mathrm{~T}_{x}$ : MXenes for electromagnetic interference shielding. ACS Nano, 2020, 14: 50085016

84 Kim H, Wang Z, Alshareef HN. Mxetronics: Electronic and photonic applications of Mxenes. Nano Energy, 2019, 60: 179-197

85 Kamysbayev V, Filatov AS, $\mathrm{Hu} \mathrm{H}$, et al. Covalent surface modifications and superconductivity of two-dimensional metal carbide MXenes. Science, 2020, 369: 979-983

86 Sun WJ, Zhao YY, Cheng XF, et al. Surface functionalization of singlelayered $\mathrm{Ti}_{3} \mathrm{C}_{2} \mathrm{~T}_{x}$ MXene and its application in multilevel resistive memory. ACS Appl Mater Interfaces, 2020, 12: 9865-9871

87 Li C, Wang K, Li J, et al. Recent progress in stimulus-responsive twodimensional metal-organic frameworks. ACS Mater Lett, 2020, 2: 779 797

88 Gao J, He M, Lee ZY, et al. A surfactant-thermal method to prepare four new three-dimensional heterometal-organic frameworks. Dalton Trans, 2013, 42: 11367-11370

89 Zhang X, Wan K, Subramanian P, et al. Electrochemical deposition of metal-organic framework films and their applications. J Mater Chem A, 2020, 8: 7569-7587

90 Wu Z, Adekoya D, Huang X, et al. Highly conductive two-dimensional metal-organic frameworks for resilient lithium storage with superb rate capability. ACS Nano, 2020, 14: 12016-12026

91 Yan Y, Li C, Wu Y, et al. From isolated Ti-oxo clusters to infinite Tioxo chains and sheets: Recent advances in photoactive Ti-based MOFs. J Mater Chem A, 2020, 8: 15245-15270

92 Li C, Wang K, Li J, et al. Nanostructured potassium-organic framework as an effective anode for potassium-ion batteries with a long cycle life. Nanoscale, 2020, 12: 7870-7874

93 Yi X, Yu Z, Niu X, et al. Intrinsically stretchable resistive switching memory enabled by combining a liquid metal-based soft electrode and a metal-organic framework insulator. Adv Electron Mater, 2019, 5: 1800655

94 Ding G, Wang Y, Zhang G, et al. 2D metal-organic framework nanosheets with time-dependent and multilevel memristive switching. Adv Funct Mater, 2019, 29: 1806637
95 Rana S, Prasoon A, Jha PK, et al. Thermally driven resistive switching in solution-processable thin films of coordination polymers. J Phys Chem Lett, 2017, 8: 5008-5014

96 Park MJ, Lee JS. Zeolitic-imidazole framework thin film-based flexible resistive switching memory. RSC Adv, 2017, 7: 21045-21049

97 Wang Z, Nminibapiel D, Shrestha P, et al. Resistive switching nanodevices based on metal-organic frameworks. ChemNanoMat, 2016, 2 67-73

98 Pan L, Ji Z, Yi X, et al. Metal-organic framework nanofilm for mechanically flexible information storage applications. Adv Funct Mater, 2015, 25: 2677-2685

99 Yoon SM, Warren SC, Grzybowski BA. Storage of electrical information in metal-organic-framework memristors. Angew Chem Int Ed, 2014, 53: 4437-4441

100 Huang $\mathrm{X}$, Zheng B, Liu Z, et al. Coating two-dimensional nanomaterials with metal-organic frameworks. ACS Nano, 2014, 8: 86958701

101 Bian G, Yin J, Zhu J. Recent advances on conductive 2D covalent organic frameworks. Small, 2021, 17: 2006043

102 Zhang $\mathrm{C}$, Li Y, Li $\mathrm{H}$, et al. Overview of electric-field-induced deposition technology in fabricating organic thin films. J Mater Chem C, 2021, 9: 374-394

103 Furukawa H, Cordova KE, O'Keeffe M, et al. The chemistry and applications of metal-organic frameworks. Science, 2013, 341: 1230444

$104 \mathrm{Xu} \mathrm{S}$, Zhang Q. Recent progress in covalent organic frameworks as light-emitting materials. Mater Today Energy, 2021, 20: 100635

105 Zhi Y, Wang Z, Zhang HL, et al. Recent progress in metal-free covalent organic frameworks as heterogeneous catalysts. Small, 2020 16: 2001070

106 Yu F, Liu W, Ke SW, et al. Electrochromic two-dimensional covalent organic framework with a reversible dark-to-transparent switch. Nat Commun, 2020, 11: 5534

107 Yao CJ, Wu Z, Xie J, et al. Two-dimensional (2D) covalent organic framework as efficient cathode for binder-free lithium-ion battery. ChemSusChem, 2020, 13: 2457-2463

108 Sun B, Li X, Feng T, et al. Resistive switching memory performance of two-dimensional polyimide covalent organic framework films. ACS Appl Mater Interfaces, 2020, 12: 51837-51845

109 Liu J, Yang F, Cao L, et al. A robust nonvolatile resistive memory device based on a freestanding ultrathin $2 \mathrm{D}$ imine polymer film. Adv Mater, 2019, 31: 1902264

110 Wang R, Mujahid M, Duan Y, et al. A review of perovskites solar cell stability. Adv Funct Mater, 2019, 29: 1808843

111 Wang N, Liu W, Zhang Q. Perovskite-based nanocrystals: Synthesis and applications beyond solar cells. Small Methods, 2018, 2: 1700380

112 Sun J, Wu J, Tong X, et al. Organic/inorganic metal halide perovskite optoelectronic devices beyond solar cells. Adv Sci, 2018, 5: 1700780

113 Said AA, Xie J, Zhang Q. Recent progress in organic electron transport materials in inverted perovskite solar cells. Small, 2019, 15: 1900854

114 Gu PY, Wang N, Wang C, et al. Pushing up the efficiency of planar perovskite solar cells to $18.2 \%$ with organic small molecules as the electron transport layer. J Mater Chem A, 2017, 5: 7339-7344

115 Gu PY, Wang N, Wu A, et al. An azaacene derivative as promising electron-transport layer for inverted perovskite solar cells. Chem Asian J, 2016, 11: 2135-2138

116 Yang JM, Choi ES, Kim SY, et al. Perovskite-related $\left(\mathrm{CH}_{3} \mathrm{NH}_{3}\right)_{3} \mathrm{Sb}_{2} \mathrm{Br}$ for forming-free memristor and low-energy-consuming neuromorphic computing. Nanoscale, 2019, 11: 6453-6461

117 Qian WH, Cheng XF, Zhou J, et al. Lead-free perovskite $\mathrm{MASnBr}_{3}$ based memristor for quaternary information storage. InfoMat, 2019, 2 : 743-751

118 Li B, Hui W, Ran X, et al. Metal halide perovskites for resistive switching memory devices and artificial synapses. J Mater Chem C, 2019, 7: 7476-7493

119 Huang Y, Zhao Z, Wang C, et al. Conductive metallic filaments dominate in hybrid perovskite-based memory devices. Sci China Mater, 2019, 62: 1323-1331

120 Yoo EJ, Lyu M, Yun JH, et al. Resistive switching behavior in organicinorganic hybrid $\mathrm{CH}_{3} \mathrm{NH}_{3} \mathrm{PbI}_{3-x} \mathrm{Cl}_{x}$ perovskite for resistive random 
access memory devices. Adv Mater, 2015, 27: 6170-6175

121 Choi J, Park S, Lee J, et al. Organolead halide perovskites for low operating voltage multilevel resistive switching. Adv Mater, 2016, 28: 6562-6567

$122 \mathrm{Hu} \mathrm{Y}$, Zhang S, Miao X, et al. Ultrathin $\mathrm{Cs}_{3} \mathrm{Bi}_{2} \mathrm{I}_{9}$ nanosheets as an electronic memory material for flexible memristors. Adv Mater Interfaces, 2017, 4: 1700131

123 Cheng XF, Hou X, Zhou J, et al. Pseudohalide-induced 2D $\left(\mathrm{CH}_{3} \mathrm{NH}_{3}\right)_{2} \mathrm{PbI}_{2}(\mathrm{SCN})_{2}$ perovskite for ternary resistive memory with high performance. Small, 2018, 14: 1703667

124 Yang L, Singh M, Shen S-W, et al. Transparent and flexible inorganic perovskite photonic artificial synapses with dual-mode operation. Adv Funct Mater, 2020, 31: 2008259

125 Kim H, Han JS, Kim SG, et al. Halide perovskites for resistive randomaccess memories. J Mater Chem C, 2019, 7: 5226-5234

126 Choi J, Le QV, Hong K, et al. Enhanced endurance organolead halide perovskite resistive switching memories operable under an extremely low bending radius. ACS Appl Mater Interfaces, 2017, 9: 30764-30771

127 Yan $\mathrm{K}$, Chen B, Hu H, et al. First fiber-shaped non-volatile memory device based on hybrid organic-inorganic perovskite. Adv Electron Mater, 2016, 2: 1600160

128 Paul T, Sarkar PK, Maiti S, et al. Multilevel programming and lightassisted resistive switching in a halide-tunable all-inorganic perovskite cube for flexible memory devices. ACS Appl Electron Mater, 2020, 2: 3667-3677

129 Das U, Nyayban A, Paul B, et al. Compliance current-dependent dualfunctional bipolar and threshold resistive switching in all-inorganic rubidium lead-bromide perovskite-based flexible device. ACS Appl Electron Mater, 2020, 2: 1343-1351

130 Lin Q, Hu W, Zang Z, et al. Transient resistive switching memory of $\mathrm{CsPbBr}_{3}$ thin films. Adv Electron Mater, 2018, 4: 1700596

131 Han JS, Le QV, Choi J, et al. Air-stable cesium lead iodide perovskite for ultra-low operating voltage resistive switching. Adv Funct Mater, 2018, 28: 1705783

132 Kim SG, Han JS, Kim H, et al. Recent advances in memristive materials for artificial synapses. Adv Mater Technol, 2018, 3: 1800457

Acknowledgements Zhang Q acknowledges the support from the starting funding of City University of Hong Kong (9380117) and the 111 Project (D20015). Li Y thanks the financial support from the National Natural Science Foundation of China (22008164), the Natural Science Foundation of Jiangsu Province (BK20190939), and the Natural Science Foundation of Jiangsu Higher Education Institutions of China (19KJB150018). This work was also supported by the Natural Science Foundation of Jiangsu Higher Education Institutions (18KJA470004), the Six Talent Peaks Project of Jiangsu Province, China (XCL-078), and Suzhou Key Laboratory for Low Dimensional Optoelectronic Materials and Devices (SZS201611).

Author contributions $\mathrm{Li} \mathrm{Y}$ and Zhang $\mathrm{C}$ wrote and revised the manuscript with support from Ma C and Zhang Q; Shi Z and Wang J polished the manuscript and organized the references. All authors contributed to the general discussion.

Conflict of interest The authors declare that they have no conflict of interest.

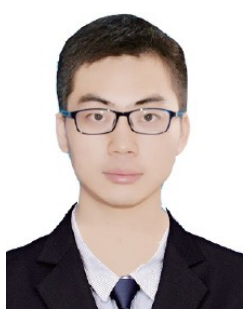

Yang Li is currently an associate professor at the School of Physical Science and Technology, Suzhou University of Science and Technology. He received his $\mathrm{BS}$ and $\mathrm{PhD}$ degrees from Soochow University. From 2016 to 2017, he studied at Prof. Qichun Zhang's group as a joint $\mathrm{PhD}$ student. His research interests focus on the preparation of novel memristive materials and devices, and their applications for data storage and neuromorphic computing.

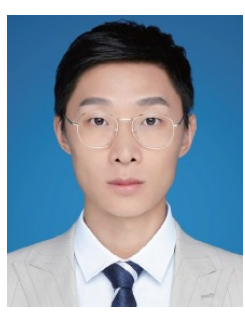

Cheng Zhang is currently a research fellow at the School of Physical Science and Technology, Suzhou University of Science and Technology. From 2019 to 2020, he studied at Prof. Qichun Zhang's group as a joint $\mathrm{PhD}$ student. His research interests involve the synthesis and application of organic semiconductor materials, particularly, organic resistive memory devices.

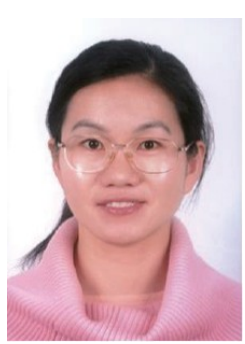

Chunlan Ma is a professor and the dean of the School of Physical Science and Technology, Suzhou University of Science and Technology. Her research interests include electronic structure, band structure investigation and the design of novel optoelectronic materials and devices using first-principles calculations.

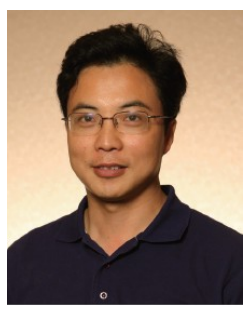

Qichun Zhang is a professor at the Department of Materials Science and Engineering, City University of Hong Kong. Before he moved to Hong Kong, he was an associate professor with tenure at the School of Materials Science and Engineering, Nanyang Technological University of Singapore. He is currently a fellow of the Royal Society of Chemistry. His research interests include carbon-rich conjugated materials and their applications for optoelectronic and semiconductor devices.

\section{基于结晶材料的柔性忆阻器的研究进展}

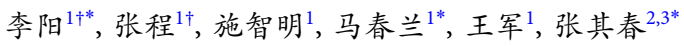

摘要 忆阻器因其结构独特以及电学行为能够模拟生物突触, 近年来 在存算一体化和人工神经网络领域显现出广阔的应用前景. 从材料多 样性的角度而言，目前已有诸多材料显示出应用于忆阻器的巨大潜力. 在本文中, 我们关注一类基于结晶材料的柔性忆阻器. 首先介绍了典型 的忆阻器件结构与开关机制，其次重点综述了基于结晶材料的柔性忆 阻器及其在数据存储和神经形态计算领域的应用, 包括二维材料、金 属有机框架材料、共价有机框架材料和钙钛矿材料. 最后, 对当前基于 结晶材料的柔性忆阻器的发展前景进行了展望. 